\title{
The effects of prebiotics on microbial dysbiosis, butyrate production and immunity in HIV-infected subjects
}

S Serrano-Villar ${ }^{1,13}$, JF Vázquez-Castellanos ${ }^{2,3,13}$, A Vallejo $^{1}$, A Latorre $^{2,3}$, T Sainz $^{4}$, S Ferrando-Martínez $^{5}$, D Rojo $^{6}$, J Martínez-Botas ${ }^{7,8}$, J del Romero ${ }^{9}, \mathrm{~N}_{\text {Madrid }}{ }^{1}, \mathrm{M}$ Leal $^{5}$, JI Mosele ${ }^{10}$, MJ Motilva ${ }^{10}$, C Barbas ${ }^{6}$, M Ferrer ${ }^{11}$, A Moya ${ }^{2,3}$, S Moreno ${ }^{1}$, MJ Gosalbes ${ }^{2,3,14}$ and V Estrada ${ }^{12,14}$

\begin{abstract}
Altered interactions between the gut mucosa and bacteria during HIV infection seem to contribute to chronic immune dysfunction. A deeper understanding of how nutritional interventions could ameliorate gut dysbiosis is needed. Fortyfour subjects, including $12 \mathrm{HIV}^{+}$viremic untreated (VU) patients, 23 antiretroviral therapy-treated (ART ${ }^{+}$) virally suppressed patients ( 15 immunological responders and 8 non-responders) and 9 HIV $^{-}$controls ( $\mathrm{HIV}^{-}$), were blindly randomized to receive either prebiotics (scGOS/lcFOS/glutamine) or placebo (34/10) over 6 weeks in this pilot study. We assessed fecal microbiota composition using deep 16S rRNA gene sequencing and several immunological and genetic markers involved in HIV immunopathogenesis. The short dietary supplementation attenuated HIV-associated dysbiosis, which was most apparent in VU individuals but less so in $\mathrm{ART}^{+}$subjects, whose gut microbiota was found more resilient. This compositional shift was not observed in the placebo arm. Significantly, declines in indirect markers of bacterial translocation and T-cell activation, improvement of thymic output, and changes in butyrate production were observed. Increases in the abundance of Faecalibacterium and Lachnospira strongly correlated with moderate but significant increases of butyrate production and amelioration of the inflammatory biomarkers soluble CD14 and high-sensitivity C-reactive protein, especially among VU. Hence, the bacterial butyrate synthesis pathway holds promise as a viable target for interventions.
\end{abstract}

\section{INTRODUCTION}

HIV has emerged as a chronic inflammatory disease in which chronic immune dysfunction leads to sustained inflammation and contributes to an increased risk of death. ${ }^{1-3}$ Several recent studies have shown that HIV infection causes dysbiosis and alters the interplay between the gut bacterial communities and the immune system, which has changed the understanding of how HIV infection influences mucosal and systemic immunity by stimulating inflammation in the gut and systemic circulation. It is known that HIV infection induces a compositional shift of gut microbiota, with enrichment of bacterial populations that are either pro-inflammatory or potentially pathogenic ${ }^{4-10}$ and changes in microbial diversity that are correlated with immune status. ${ }^{11}$ This perturbation of the gut ecosystem might go far beyond the compositional level and reach bacterial functional

${ }^{1}$ Department of Infectious Diseases, Hospital Universitario Ramón y Cajal, Facultad de Medicina, Universidad de Alcalá (IRYCIS), Madrid, Spain. ${ }^{2}$ Unidad Mixta de Investigación en Genómica y Salud de la Fundación para el Fomento de la Investigación Sanitaria y Biomédica de la Comunidad Valenciana (FISABIO-Salud Pública) y el Instituto Cavanilles de Biodiversidad y Biología Evolutiva (Universidad de Valencia), Valencia, Spain. ${ }^{3}$ CIBER en Epidemiología y Salud Pública (CIBERESP), Madrid, Spain. ${ }^{4}$ Department of Pediatric Infectious Diseases, University Hospital La Paz, and La Paz Research Institute, Madrid, Spain. ${ }^{5}$ Laboratory of Immunovirology, Biomedicine Institute of Seville (IBIS), Department of Infectious Diseases, University Hospital Virgen del Rocío, Sevilla, Spain. ${ }^{6}$ Centro de Metabolómica y Bioanálisis (CEMBIO), Facultad de Farmacia, CEU San Pablo University, Madrid, Spain. ${ }^{7}$ Department of Biochemistry, University Hospital Ramón y Cajal-IRYCIS, Madrid, Spain. ${ }^{8} \mathrm{CIBER}$ of Obesity and Nutrition Pathophysiology (CIBEROBN), Madrid, Spain. ${ }^{9}$ Centro Sandoval, Madrid, Spain. ${ }^{10}$ Food Technology Department, Agrotecnio Center, University of Lleida, Spain. ${ }^{11}$ Institute of Catalysis, Consejo Superior de Investigaciones Científicas (CSIC), Madrid, Spain and ${ }^{12}$ HIV Unit, Department of Internal Medicine, University Hospital Clínico San Carlos, Madrid, Spain. Correspondence: S Serrano-Villar (sergio.serrano@salud.madrid.org) or MJ Gosalbes (Maria.Jose.Gosalbes@uv.es)

${ }^{13}$ These authors contributed equally to this work.

${ }^{14}$ These are co-senior authors in this work. 
capabilities, with enrichment of genes involved in various pathogenic processes and inflammatory pathways ${ }^{9}$ and production of metabolites that directly influence host health, such as the immunomodulatory derivatives kynurenine ${ }^{12}$ and shortchain fatty acids (SCFAs). ${ }^{13-15}$ SCFAs are end products of bacterial anaerobic fermentation of dietary fiber and might influence host health by different mechanisms. In particular, butyrate is considered the preferred energy source of colon epithelial cells because $70 \%$ of their energy is derived from the oxidation of this acid. ${ }^{16}$ This metabolite activates genes involved in different pathways, such as colonic gluconeogenesis, or genes responsible for the proliferation and differentiation of epithelial cells. ${ }^{17}$ Moreover, butyrate has been shown to contribute to maintaining homeostasis in the gut by promoting immunotolerance to commensal bacteria via the downregulation of lipopolysaccharide-induced proinflammatory mediators. ${ }^{13}$

Although different studies in HIV patients ${ }^{18-23}$ have described changes in immunity and in intestinal bacteria after prebiotic, probiotic, or symbiotic administration, a deeper understanding of the ecological effects of such interventions on the structure and function of gut bacterial communities is needed, especially in the context of HIV-infected individuals who are undergoing treatment. In HIV-infected individuals not receiving antiretroviral therapy (ART), interventions with probiotics and/or prebiotics or a mixture (symbiotics) have been shown to contribute to the maintenance of immune functions over a longer period. ${ }^{21,24,25}$ These therapies included fructo-oligosaccharides (FOS $)^{24}$, mixtures of galacto-oligosaccharides (GOS) and polyunsaturated acids ${ }^{21}$, and the bacterial strains Lactobacillus rhamnosus, Lactobacillus reuteri and Bifidobacterium lactis Bi-07. ${ }^{24,25}$ Gori et al. demonstrated, in a controlled study of ART-naive HIV-infected subjects, significant declines of markers of inflammation (soluble CD14) and T-cell activation (\%CD4 ${ }^{+} \mathrm{CD} 25^{+}$T-cells) in association with increased bifidobacteria and decreased pathogenic Clostridium lituseburense and Clostridium histolyticum levels. ${ }^{18}$ Subsequently, in SIV-infected macaques beginning ART, a symbiotic treatment (a mixture of prebiotics and probiotics) resulted in increased frequency and functionality of gastrointestinal antigen-presenting cells, enhanced reconstitution and functionality of $\mathrm{CD}^{+}$T-cells, and reduced fibrosis of lymphoid follicles in the colon. ${ }^{26}$ Recently, in two placebo-controlled trials with treated HIV-infected individuals, a short probiotic intervention elicited a significant reduction in inflammatory predictors of mortality, i.e., interleukin-6 and D-dimers. ${ }^{22,23}$ Outside the field of HIV infection, glutamine has been shown to be a crucial metabolic fuel source for enterocytes, enhancing cell turnover, attenuating bacterial translocation and, conceivably, altering gut microbiota structure. $^{27-29}$

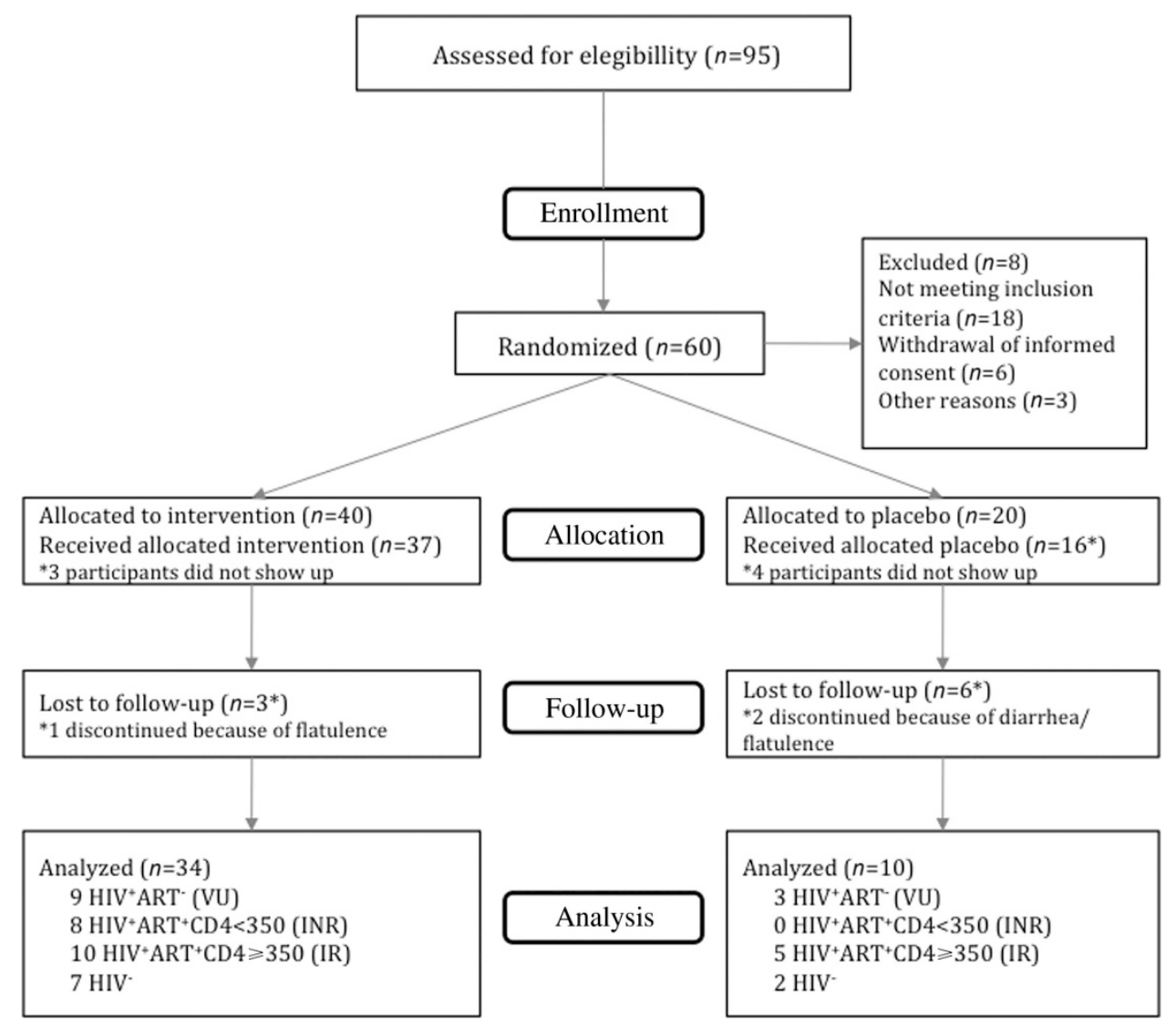

Figure 1 Study profile. INR, immunological non-responder; IR, immunological responder; VU, viremic untreated. 
We hypothesized that dietary supplementation with prebiotics and glutamine, which have shown beneficial effects on gut microbiota ${ }^{30}$ and the enterocyte barrier function, ${ }^{31}$ might ameliorate HIV-associated dysbiosis and improve innate and adaptive immunity. In this pilot study, we determined for the first time the SCFA profile of the intestinal bacteria in HIVinfected individuals, and we assessed the interactions among these immunomodulatory derivatives, the microbiota and immunological markers of disease progression after dietary supplementation with prebiotics.

\section{RESULTS}

\section{General characteristics of the study population and safety} data

A total of 44 individuals completed the 6-week course of treatment, with 34 receiving prebiotics and 10 receiving a placebo. These included 12 viremic, ART-untreated (VU) HIVinfected subjects, 15 immunological ART responders (IR), 8 non-responders (INR), and $9 \mathrm{HIV}$-uninfected $\left(\mathrm{HIV}^{-}\right)$controls (Figure 1). HIV-infected individuals on ART were representative of a medium-aged population undergoing long-term treatment without comorbidities (Table 1). No statistically significant differences were observed among the groups in dietary habits (Supplementary Figure S1 online). The average compliance to the intervention in both arms was superior to $85 \%$, and overall, the intervention was well tolerated (see Supplementary Table S1 online for a description of adverse events).

\section{Effects of the nutritional prebiotic intervention on gut microbiota structure}

Alpha diversity is used to measure the richness and evenness of bacterial taxa within a community. We found that, before intervention, $\mathrm{VU}$ subjects had the highest microbial richness of the cohort for each of the four different metrics (ACE, Chaol, Shannon, and the total of observed species), followed by HIV ${ }^{-}$ individuals, while INR subjects showed the lowest diversity (Figure 2a and Supplementary Table S2). The nutritional intervention did not result in a significant variation of the alpha diversity parameters, as the microbiota of VU individuals was still the most diverse at the end of the study, whereas that of INR individuals remained the least diverse (Figure 2a and Supplementary Table S3). Analysis of changes in betadiversity showed that the prebiotic intervention significantly modified the microbiota structure in all the groups (Figure $\mathbf{2 b}$ and Supplementary Tables S4 and S5).

Then, we asked whether the differences in the gut microbiota before and after the treatment might be representative of the disease status. Before treatment, partitioning around medoids analysis revealed distinct clustering in the subject groups, representing the extremes of health and disease, the $\mathrm{HIV}^{-}$and $\mathrm{HIV}^{+}$individuals (Supplementary Figure S2A online; ADONIS; $P=0.001$ ). In addition, all $\mathrm{HIV}^{+}$individuals harbored a dysbiotic microbiota compared with $\mathrm{HIV}^{-}$ individuals (Supplementary Figure S2; ADONIS, $P=0.001$ ). Given the recent evidence that HIV-associated dysbiosis might be explained by sexual practices, ${ }^{32}$ we

Table 1 Characteristics of the study population

\begin{tabular}{|c|c|c|c|c|c|}
\hline & $\begin{array}{l}\mathrm{HIV}^{+} \mathrm{ART}^{-}(\mathrm{VU}) \\
(N=12)\end{array}$ & $\begin{array}{c}\mathrm{HIV}^{+} \mathrm{ART}^{+} \mathrm{CD} 4<350 \text { (INR) } \\
(N=8)\end{array}$ & $\begin{array}{c}\mathrm{HIV}^{+} \mathrm{ART}^{+} \mathrm{CD} 4 \geqslant 350(\mathrm{IR}) \\
(N=15)\end{array}$ & $\begin{array}{l}\mathrm{HIV}^{-} \\
(\boldsymbol{N}=9)\end{array}$ & $\boldsymbol{P}$ value \\
\hline Age (years) & $34(33-35)$ & $48(41-53)$ & $40(33-48)$ & $47(31-60)$ & 0.096 \\
\hline CD4 + T-cell count (cells mm ${ }^{-3}$ ) & $558(432-646)$ & $291(230-324)$ & $561(426-794)$ & $762(653-878)$ & $<0.001$ \\
\hline CD4/CD8 ratio & $0.53(0.44-0.65)$ & $0.71(0.35-0.89)$ & $1.13(0.96-1.26)$ & $1.51(1.20-1.78)$ & $<0.001$ \\
\hline Time since HIV diagnosis (years) & $5.4(2.9-9.9)$ & $11.8(5.6-20.8)$ & $8.0(4.5-13.9)$ & - & 0.124 \\
\hline Duration of ART (years) & - & $8.9(4.6-12.7)$ & $6.1(4.3-11.7)$ & - & 0.560 \\
\hline Framingham Risk Score (\%) & $2(1-2.5)$ & $4(4-7)$ & $4(1-7)$ & $2(1-4)$ & 0.134 \\
\hline HDL cholesterol $\left(\mathrm{mg} \mathrm{dl}^{-1}\right)$ & $47(42-54)$ & $50(43-60)$ & $54(50-61)$ & $62(48-75)$ & 0.020 \\
\hline LDL cholesterol (mg dl ${ }^{-1}$ ) & $89(87-102)$ & $78(67-126)$ & $112(92-124)$ & $97(75-133)$ & 0.046 \\
\hline Active arm (No., \%) & $9(75)$ & $8(100)$ & $10(62.5)$ & $7(77.8)$ & 0.682 \\
\hline
\end{tabular}

Abbreviations: ART, antiretroviral therapy; HIV ${ }^{-}$, HIV-uninfected healthy controls; INR, HIV-infected patients immunological non-responders to antiretroviral therapy; IR, HIVinfected patients immunological responders to antiretroviral therapy; VU, HIV-infected subjects viremic untreated.

All values are expressed as P50 (P25-P75) unless otherwise expressed.

$P$ values were calculated using the Kruskal-Wallis to compare median values across all groups and the Mann-Whitney test was used to compare the values between two groups.

apercentages are calculated over the total number of subjects in each group. 
a
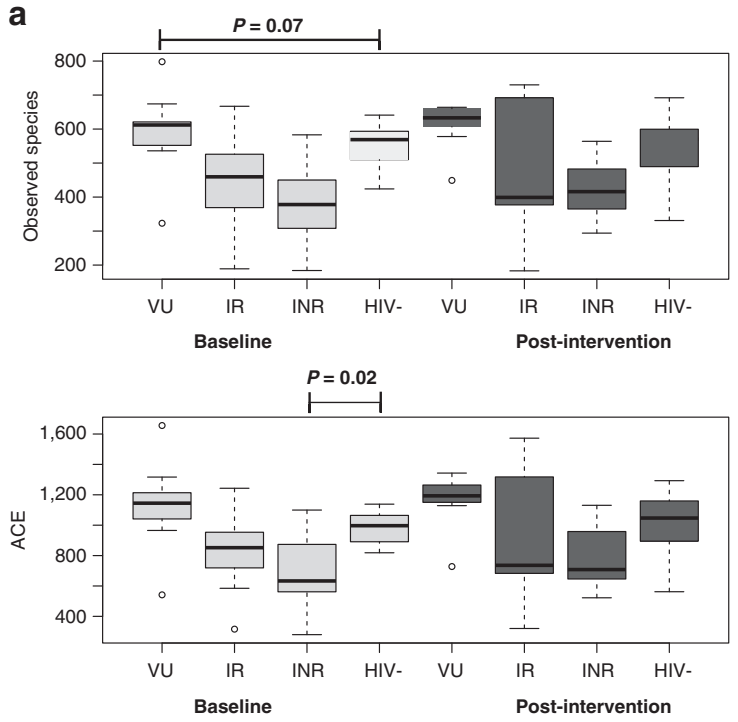
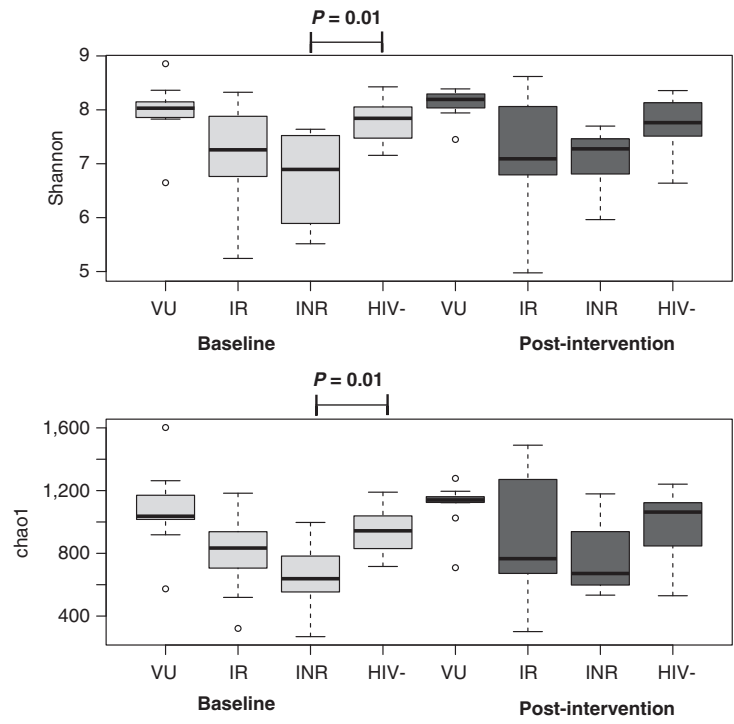

b

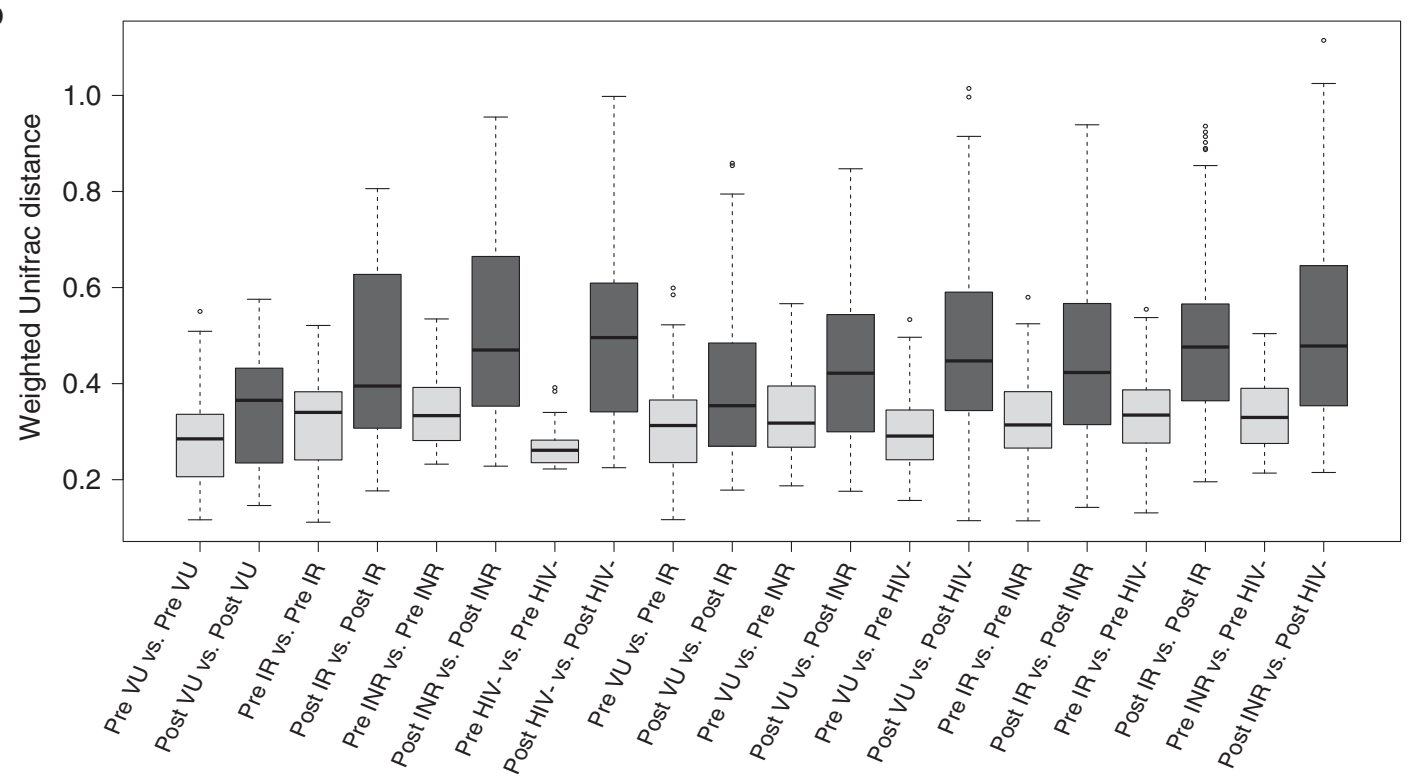

Figure 2 Diversity parameters of microbiota. (a) Alpha diversity at baseline and after prebiotics. (b) Beta diversity parameters of microbiota at baseline and after the prebiotic intervention. HIV ${ }^{-}$, HIV-uninfected control; INR, immunological non-responder, IR, immunological responder; VU, viremic untreated.

performed a permutational multivariate analysis to simultaneously adjust for this covariate. In keeping with recent observations, sexual orientation appeared as an environmental influence on microbiota composition, explaining $9 \%$ of the variance in the microbiota composition across groups $(P<0.001)$. However, this effect was weaker than the impact elicited by HIV itself, which explained $12 \%$ of the microbiota variability $(P<0.001)$ after adjusting for sexual orientation (Supplementary Table S6).

Unsupervised clustering analyses revealed that although the intervention did not cause the bacterial communities in IR individuals to approximate those of the $\mathrm{HIV}^{-}$group, VU individuals and, to a lesser extent, INR individuals experienced a compositional shift towards the control group (Figure 3). Among the patients receiving the placebo, only one migrated to the $\mathrm{HIV}^{-}$cluster, indicating that the observed alterations were attributable to the prebiotic intervention.

We further investigated which genera determined baseline divergences of microbial communities across groups using the LEfSe biomarker discovery tool (Figure $\mathbf{4}$ and Supplementary Figure S3A). As observed in previous studies, ${ }^{4,6,8,10,33}$ before the intervention, Prevotella copri was the most enriched species in all three groups of $\mathrm{HIV}^{+}$subjects (Supplementary Figure S3A). Other genera in the Firmicutes phylum, including Eubacterium, Acidaminococcus, and Mitsuokella, were consistently enriched. In contrast, the most depleted genus was Bacteroides. Faecalibacterium was also consistently depleted among $\mathrm{HIV}^{+}$individuals (Figure $\mathbf{4}$ and Supplementary Figure S3A-C). This genus includes Faecalibacterium prausnitzii, an important butyrate 

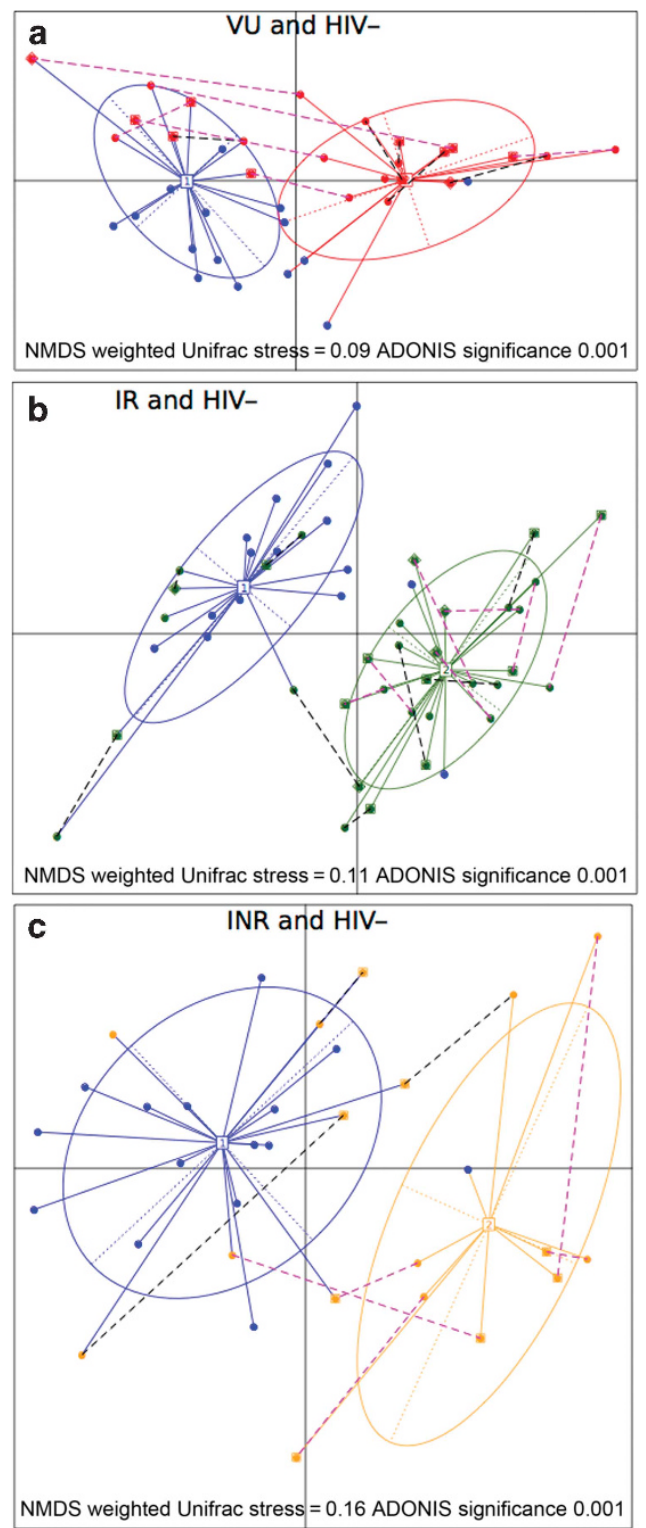

Figure 3 Changes in gut bacterial communities of each group in response to the intervention (prebiotic and placebo arms). Nonmetric Multidimensional Scaling (NMDS) analysis of the composition distribution at OTU level based on the weighted Unifrac distance matrix. (a) VU (red dots) vs. HIV ${ }^{-}$at baseline (blue dots). (b) IR (green dots) vs. $\mathrm{HIV}^{-}$at baseline (blue dots). (c) INR (orange dots) vs. $\mathrm{HIV}^{-}$at baseline (blue dots). Each point is connected with a dash line to another dot corresponding to the sample at the end of follow-up. Purple dash lines indicated those samples that significantly migrated from their baseline species profile, black dash lines indicated those samples that did not significantly change their baseline species profile based on the weighted Unifrac significance test. Dots framed by a rectangle represent individuals after treatment in the active arm; dots framed by a diamond represent individuals after treatment in the placebo arm. Centroids for $\mathrm{HIV}^{-}$ individuals are represented as " 1 " and those for $\mathrm{HIV}^{+}$patients are represented as " 2 ". Ellipses represent $70 \%$ of the samples belonging to each condition. Community differences were calculated using ADONIS test. INR, immunological non-responder; IR, immunological responder; $\mathrm{VU}$, viremic untreated.

producer in the human gut that has anti-inflammatory effects and induces the novel immunoregulatory $\mathrm{T}$-cell subset CD4CD8 $\alpha \alpha^{34}$ LEfSE analysis confirmed the depletion of
Faecalibacterium prausnitzii in the fecal microbiota of $\mathrm{HIV}^{+}$ individuals and the depletion of other butyrate-producing genera, such as Lachnospira, Anaerostipes, Butyricimonas, Coprococcus (Supplementary Figure S3A) and Roseburia (Figure 4). ${ }^{14}$ When we evaluated which specific species' abundance differed after the prebiotic treatment, microbiota variation was apparent only in the VU individuals (Figure 5), with an increase in Firmicutes (Faecalibacterium, Catenibacterium, Blautia, Eubacterium) and Actinobacteria (Collinsella and Corinebacterium).

Taken together, alpha diversity analyses showed that the number of taxa is not influenced by the dietary intervention. However, the intervention significantly modified microbiota structure, as shown by changes in beta diversity (Figures 2 and 3). Interestingly, compared with healthy controls, the VU group treated with the prebiotic showed attenuated HIV-associated dysbiosis and increased growth of beneficial genera, which was not observed among ART-treated patients (Figure 5).

SCFA profile of HIV-dysbiotic bacteria and effects of the intervention

The above analysis implicated butyrate bacterial producers as potential key bacteria in HIV immunopathogenesis. To provide mechanistic insights into the influence of the altered bacterial communities on the host's health, we measured the abundance of SCFAs, including acetate, propionate, butyrate, valerate, isobutyrate, and isovalerate, in fecal bacteria (Figure 6). At baseline, a different SCFA profile characterized the HIVinfected group (Supplementary Figure S4; ADONIS, $P=0.019)$ with more abundant propionate and lower levels of acetate ( $P=0.05$ and 0.036 , respectively) and no significant variation in the other four acids, including butyrate. After the prebiotic intervention, the propionate abundance increased in HIV-uninfected individuals, and only butyrate significantly increased in VU individuals (Figure 7, $P=0.05$ ). It should be noted that among all of the SCFAs, butyrate seems to exert the most profound effects on gut health. ${ }^{35}$

The bacterial species whose abundance changed in response to the prebiotic intervention (Figure 5) are salient candidates to be directly, or in association with a third species, related to the metabolism of the SCFAs. Hence, we examined whether shifts in species abundance determined changes in SCFA abundance. We found that butyrate abundance correlated positively with butyrate-producer species, including Roseburia faecis ( $P=0.0003)$, Lachnospira $(P=0.0016)$, Ruminococcus torques $(P=0.0093)$ and Faecalibacterium prausnitzii $(P=0.0121$; Supplementary Table S7).

\section{Changes in plasma biomarkers of activation of innate immunity}

Given that a number of bacterial species have been suggested to enhance pro-inflammatory pathways during chronic HIV infection ${ }^{8-10}$ and that structural changes of the epithelial barrier persist even during successful ART, ${ }^{36}$ we hypothesized that an intervention aimed at restoring the gut microbiota and enhancing the gut barrier would affect markers 

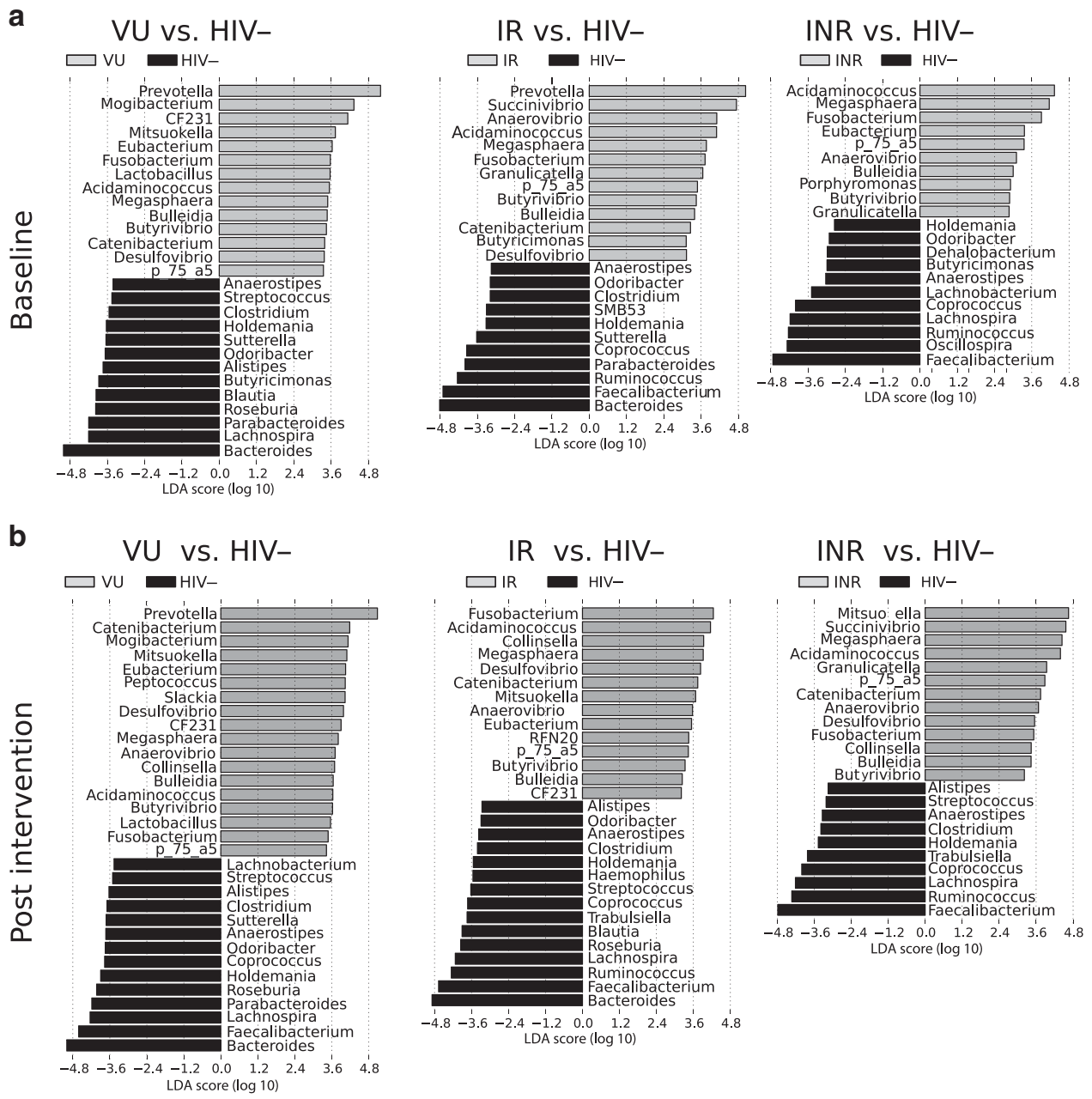

Figure 4 Bacterial taxa driving HIV-associated dysbiosis using LEfSe analysis in each group. (a) At baseline. (b) After the prebiotic intervention. The linear discriminative analysis (LDA) scores (log 10) for the most prevalent taxa among $\mathrm{HIV}^{+}$individuals are represented in the positive scale, whereas LDA-negative scores indicate those taxa enriched in $\mathrm{HIV}^{-}$individuals at baseline. $\mathrm{HIV}^{-}$, HIV-uninfected controls; INR, immunological non-responders; $\mathrm{IR}$, immunological non-responders; VU, viremic untreated.

of innate immunity activation, i.e., markers involved in inflammatory signaling (interleukin-6, high-sensitivity C-reactive protein (hs-CRP)), D-dimers indicating thrombosis, asymmetric dymethilargininine indicating vascular dysfunction and soluble CD14 and bactericidal permeability-increasing protein (BPI) indicating bacterial translocation; Figure 8a). No significant decreases were observed after the intervention, except for the BPI levels. This protein is produced by neutrophils and epithelial cells in response to pro-inflammatory stimuli and neutralizes the lipopolysaccharide. Hence, it is considered an indirect marker of bacterial translocation and a predictor of severe atherosclerosis. ${ }^{37}$ The BPI levels decreased in all the groups to the levels observed at baseline in the $\mathrm{HIV}^{-}$ group (Delta change: VU, $-24 \pm 11, P=0.154$; INR, $-21 \pm 14, P=0.074$; IR, $-56 \pm 24, P=0.009$ ).

\section{Changes in plasma trimethylamine n-oxide (TMAO) concentrations}

Metabolism of dietary choline by gut bacterial communities is a pathway with the potential to promote atherosclerosis in
non-HIV-infected patients by producing proatherogenic TMAO ${ }^{38}$ After the prebiotic treatment, we found no significant differences in the median TMAO concentrations at baseline among groups, and a significant increase was noted in the IR group (Delta change: VU, $1.2 \pm 0.6, P=0.298$; INR, $-0.2 \pm 0.9, P=0.803 ; \quad \mathrm{INR}, 7.3 \pm 0.5, P<0.001 ; \mathrm{HIV}^{-}$, $18.5 \pm 16.6, P=0.134$; Figure 8a).

\section{Changes in markers of activation of adaptive immunity}

HIV-associated dysbiosis has also been linked to activation of adaptive immunity, which can predict disease progression during ART. ${ }^{39}$ Hence, we evaluated changes in T-cell activation markers (HLA-DR ${ }^{+} \mathrm{CD} 38^{+}$and $\mathrm{CD} 25^{+}$) and senescence $\left(\mathrm{CD} 57^{+}\right)$. At baseline, T-cell activation markers were higher in HIV-infected patients than in healthy individuals and decreased after the intervention, especially the percentage of HLA-DR ${ }^{+} \mathrm{CD} 38^{+} \mathrm{CD} 4^{+} \mathrm{T}$ cells in the VU and IR groups (Delta change: $\mathrm{VU},-1.6 \pm 0.7, P=0.006$; INR, $-0.2 \pm 0.5$, $P=0.552 ; \quad \mathrm{IR}, \quad-0.4 \pm 0.2, \quad P=0.040 ; \mathrm{HIV}^{-}, \quad 0.2 \pm 0.2$, $P=0.402)$, the percentage of $\mathrm{HLA}-\mathrm{DR}^{+} \mathrm{CD} 38^{+} \mathrm{CD} 8^{+}$ 
a

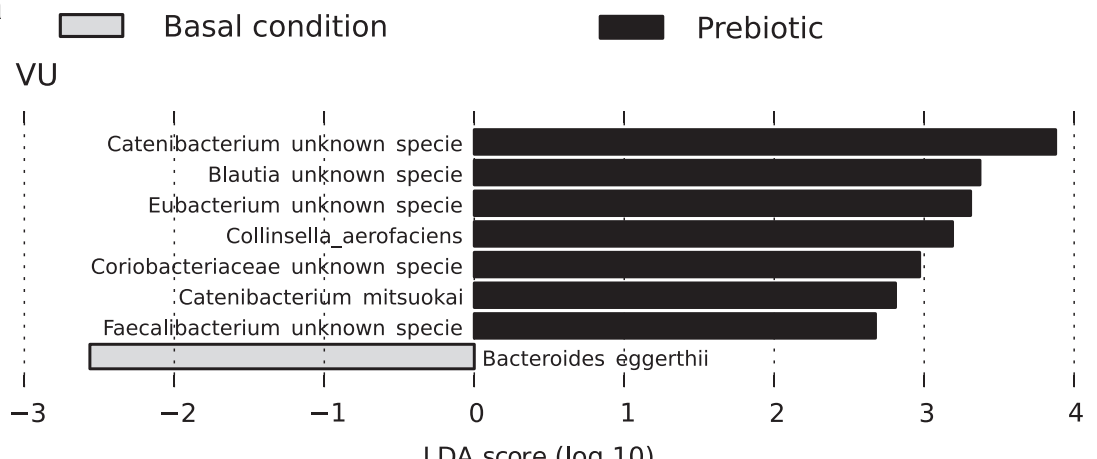

b IR

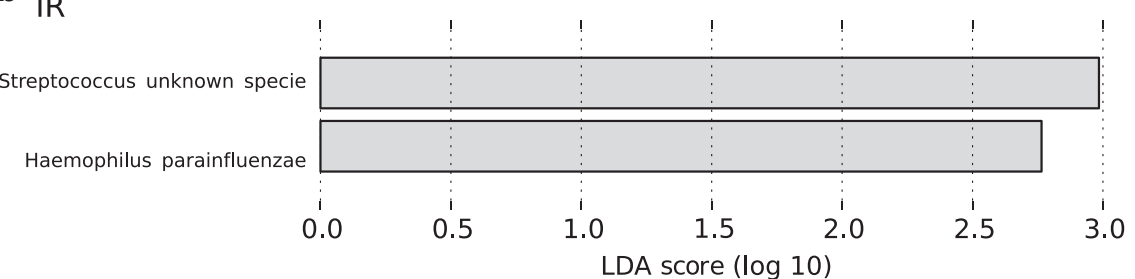

c INR

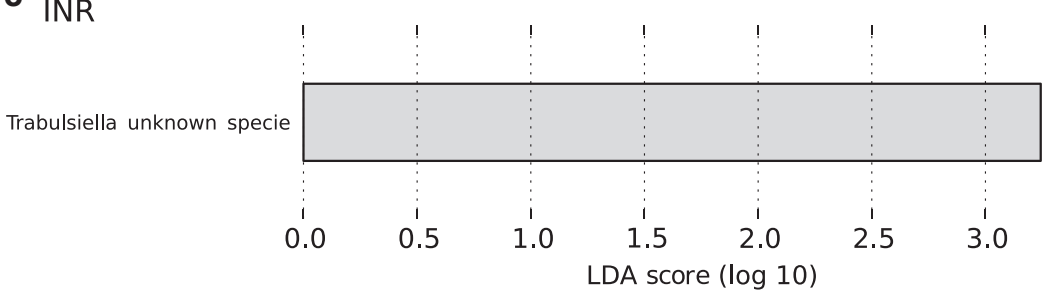

d HIV-

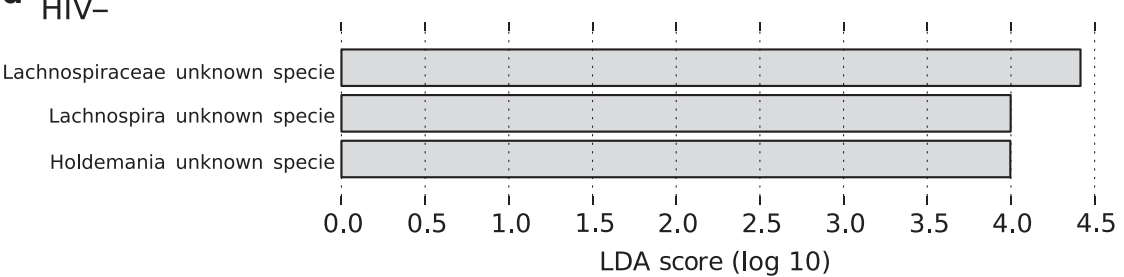

Figure 5 Changes in bacterial communities after the prebiotic intervention using LEfSe biomarker discovery tool at baseline and after the prebiotic intervention. We used the linear discriminative analysis (LDA) effect size (LEfSE) algorithm between the basal condition and the prebiotic administration into the (a) viremic untreated group (VU); (b) immunological responder group (IR); (c) immunological non-responder group (INR); (d) uninfected group $\left(\mathrm{HIV}^{-}\right)$. LDA scores $(\log 10)(\log 10 \mathrm{LDA}>2)$ for the most prevalent taxa in the basal samples are represented in the positive scale, whereas the prebiotic related species are represented in the negative scale. This analysis identified dacterial biomarkers after the prebiotic intervention only in the VU group, indicating that the intervention promoted the growth of determined bacteria in this group.

(Delta change: VU, $-3.2 \pm 1.5, P=0.015 ; \mathrm{INR}, 0.1 \pm 0.7$, $\left.P=0.788 ; \mathrm{IR}, 0.5 \pm 0.5, P=0.533 ; \mathrm{HIV}^{-},-0.5 \pm 0.3, P=0.104\right)$ and the percentage of $\mathrm{CD} 25^{+} \mathrm{CD} 8{ }^{+} \mathrm{T}$ cells (Delta change: VU, $-0.4 \pm 0.7, \quad P=0.015 ; \quad$ INR, $-0.4 \pm 0.3, P=0.646 ; \quad$ IR, $-0.05 \pm 0.8, P=0.854$; $\left.^{-} \mathrm{HIV}^{-}, 0.1 \pm 0.2, P=0.821\right)$ in VU. In contrast, we did not observe changes in the percentage of $\mathrm{CD}_{57}{ }^{+} \mathrm{T}$ cells (Figure $\mathbf{8 b}$ ). Impaired thymic function is a hallmark of immunosenescence and is associated with a proinflammatory state and poor immunological recovery during treated HIV infection. ${ }^{40}$ We studied thymic output by measuring levels of $\mathrm{T}$-cell receptor rearrangement excision circles (the TREC ratio). Overall, after treatment, thymic output improved in all groups of $\mathrm{HIV}^{+}$individuals, although it remained impaired compared with that in the $\mathrm{HIV}^{-}$controls, and the increase only reached statistical significance in $\mathrm{VU}$ individuals (Delta change: VU, $61 \pm 51, P=0.002$; INR, $47 \pm 38, P=0.179 ; \mathrm{IR}, 51 \pm 47, P=0.827 ; \mathrm{HIV}^{-}, 60 \pm 78$, $P=0.748$; Figure $8 \mathbf{b}$ ).

\section{The intervention affected RNA expression of several human genes involved in pathogenic pathways}

Mechanistically, gut microbiota might influence host health by a number of complex host-microbiota interactions. Butyrate is a potent inhibitor of histone deacetylases and is thus able to induce downstream epigenetic regulation of gene expression. ${ }^{41} \mathrm{We}$ speculated that variation in systemic 

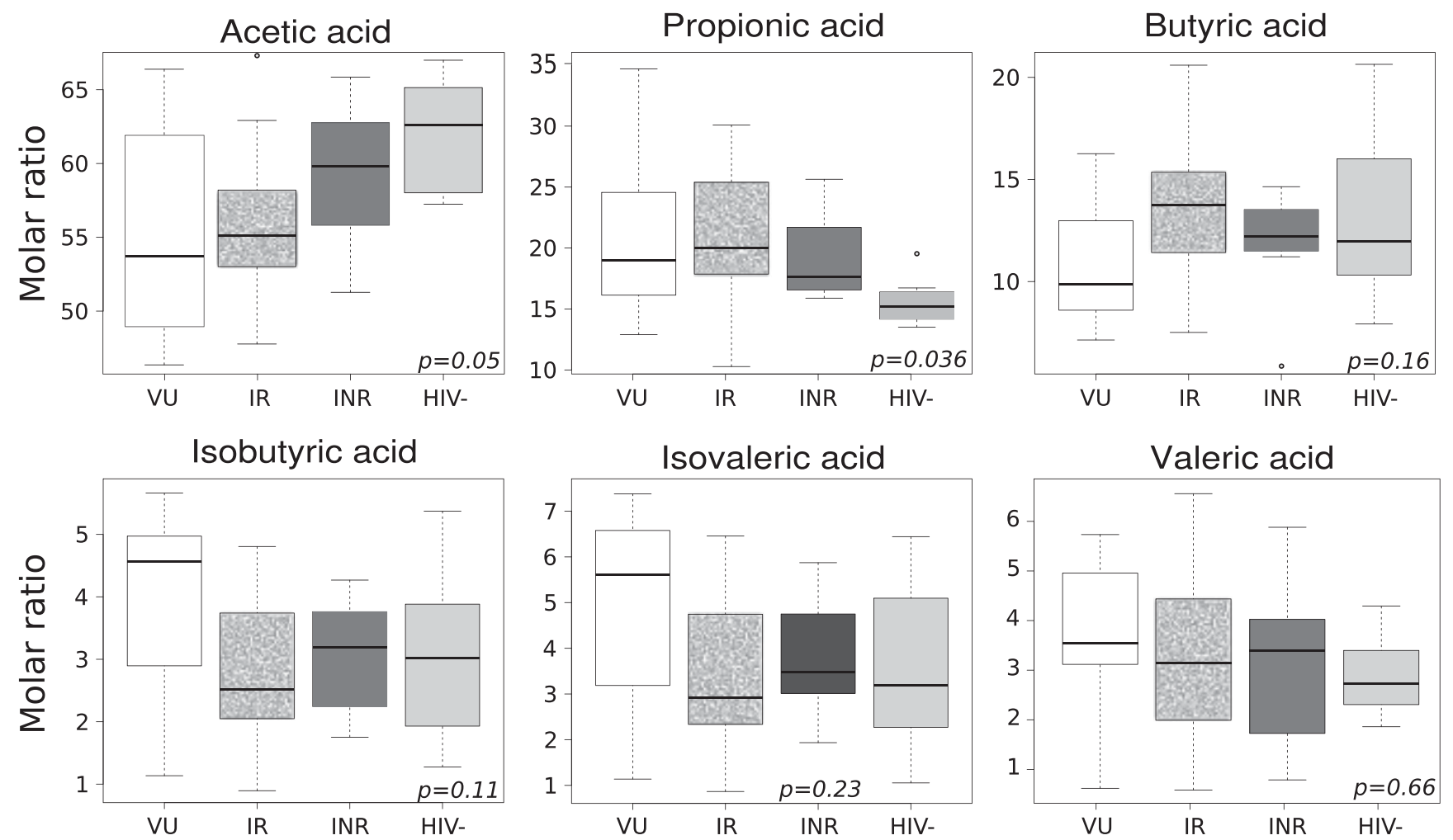

Figure 6 Relative abundance of SCFA in fecal bacteria per groups. The $P$ value reflects the differences in the relative abundance for the grouped comparison using a Kruskal-Wallis test. The concentration is expressed as millimolar of SCFA. INR, immunological non-responder, IR, immunological responder; SCFA, short-chain fatty acid; VU, viremic untreated.

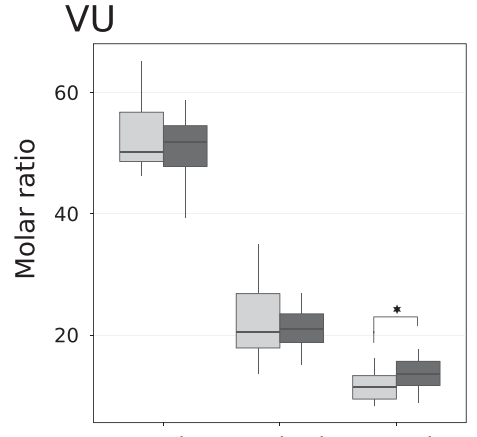

Acetic Propionic Butyric

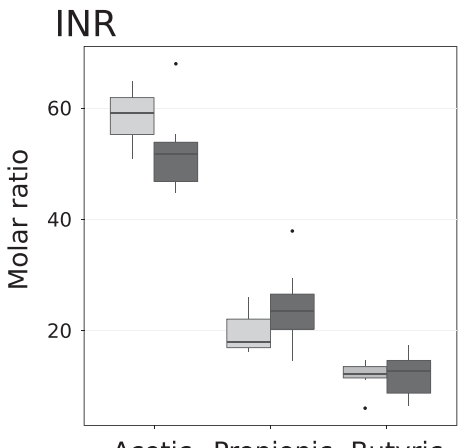

Acetic Propionic Butyric
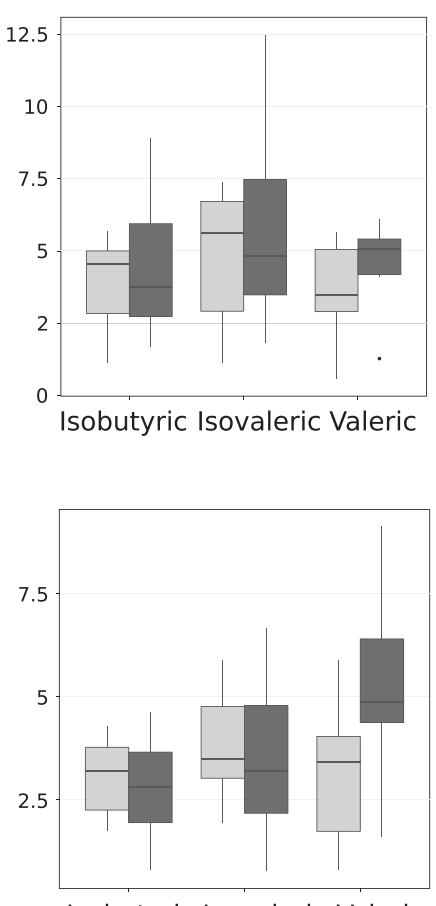

Isobutyric Isovaleric Valeric

Baseline
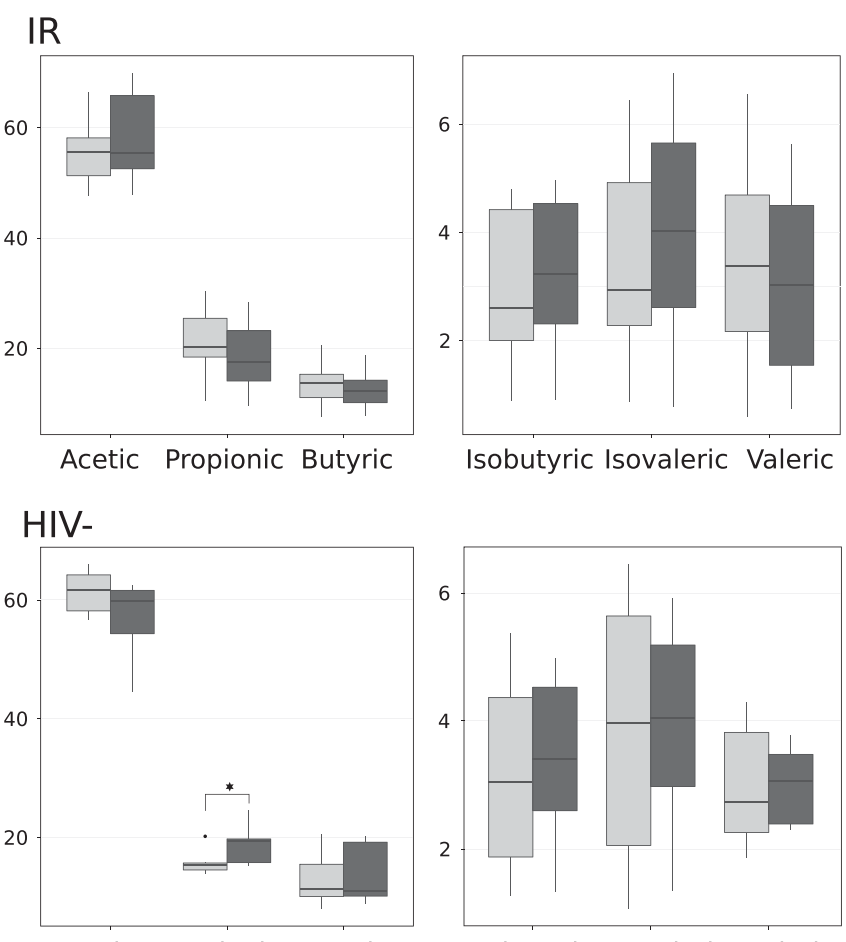

Acetic Propionic Butyric

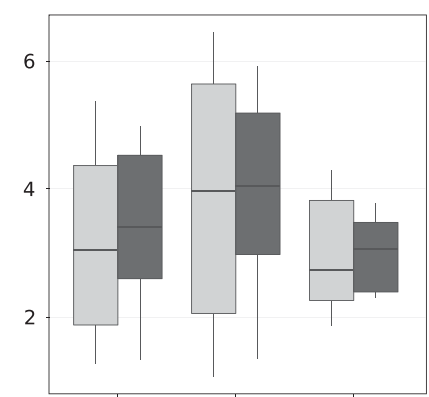

Isobutyric Isovaleric Valeric

After prebiotic

Figure 7 Relative abundance of SCFA in fecal bacteria before (orange) and after (yellow) the prebiotic intervention. To calculate differences across groups in continuous variables, we used the Kruskal-Wallis test; and for differences between groups, we used Wilcoxon rank-sum tests. The concentration is expressed as millimolar of SCFA. INR, immunological non-responder, IR, immunological responder; SCFA, short-chain fatty acid; VU, viremic untreated. 
a
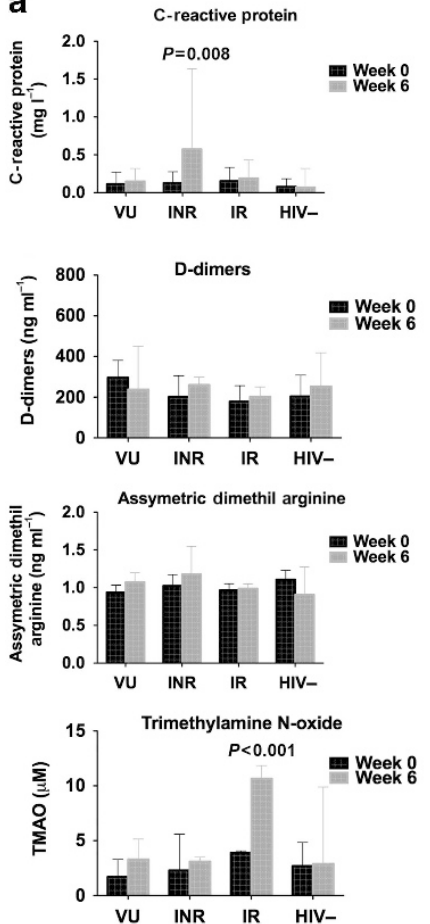

b
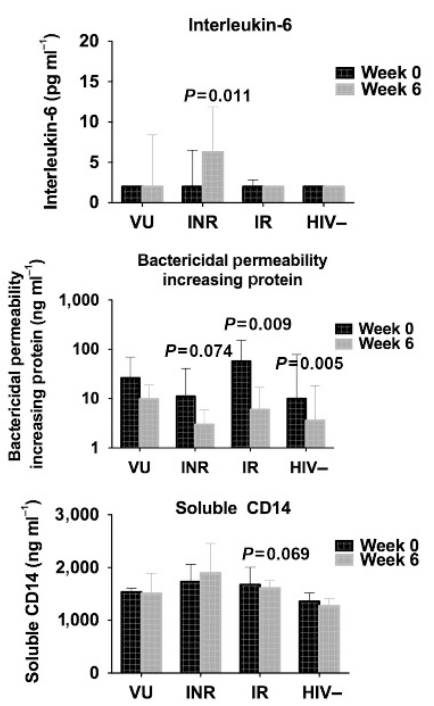
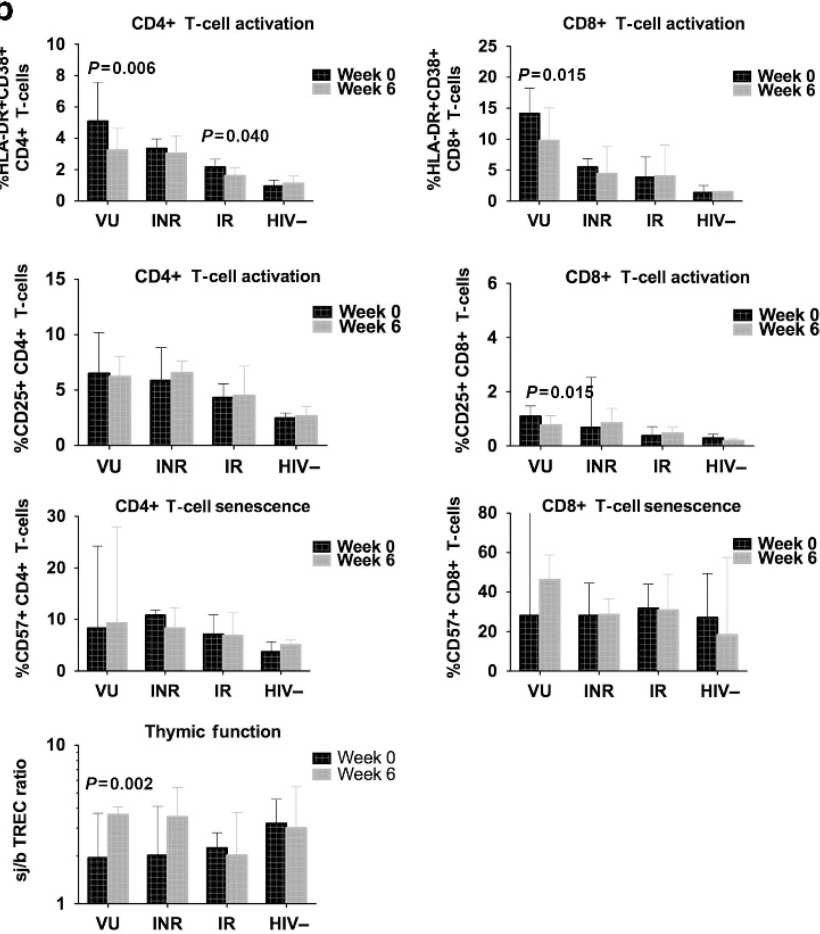

Figure 8 (a) Changes in systemic inflammatory markers in the prebiotic arm. (b) Changes in systemic markers of immune activation and thymic output in the prebiotic arm. INR, immunological non-responder, IR, immunological responder; VU, viremic untreated.

markers might be explained by downstream regulation of genes involved in HIV immunopathogenesis. We assessed a number of genes involved in cell cycle regulation (TP53), the innate and adaptive immune response (APOBEC3G, CCL2, CCR2, CXCL10, IFNA1), HIV-mediated cell activation (BCL2, STAT1), immune exhaustion (PDCD1), reverse cholesterol transport (SCARB1) and fatty acid storage and glucose metabolism (PPARG) (Supplementary Figure S5). After the intervention, we detected significant changes in the expression of some genes in all the groups except the IR group. In VU and INR subjects, we observed upregulation of CCR2, a gene involved in enhanced trafficking of blood monocytes to the gut, ${ }^{42}$ and TP53, which encodes the p53 tumor-suppressor protein. In VU individuals, the expression of CXCL10, encoding an interferon-gammainduced chemokine that mediates the recruitment of T-cells and NK cells, ${ }^{43}$ was also significantly upregulated.

\section{Potential microbial targets for treatment of immune dysfunction in patients}

The above analyses helped us identify taxa that might ameliorate the sustained proinflammatory state characteristic of HIV infection through the butyrate synthesis pathway and are thus of therapeutic interest. To untangle the impact of the prebiotic intervention on the complex interactions among key microbiota components, butyrate production, and circulating immunological predictors of disease progression, we speculated that the observed effects on systemic markers may be a consequence of changes in a subset of key bacteria, which could serve as potential microbial targets for interventions. Hence, we investigated whether changes in genera contributing to
$\mathrm{HIV}$-associated dysbiosis were associated with significant differences in SCFA abundance and peripheral markers of disease progression using generalized linear models and Bayesian networks (Figure 9 and Supplementary Table S8). First, the analysis suggested that the disturbances in some of the taxa driving HIV-associated dysbiosis might contribute to maintenance of persistent immune dysfunction, while changes in the abundance of other genera seemed a mechanism to alleviate it (Figure 9a). For example, Bacteroides was the most depleted genus in VU and IR (Figure 3 and Supplementary Figure S4), and their decrease strongly correlated with an increase of T-cell activation (Figure 9a). Similarly, Prevotella and Acidaminococcus were among the most enriched genera in $\mathrm{HIV}$-infected individuals, and their increase further led to increased T-cell activation (Figures 4 and 9a). Hence, Bacteroides, Prevotella, and Acidaminococcus represent central components of the HIV-associated dysbiosis that seems to contribute to chronic immune dysfunction. In contrast, other taxa followed the reverse pattern of associations between their abundance at baseline and the effects of their changes on peripheral markers, likely representing a beneficial adaptation of the gut ecosystem. For example, all the groups of HIV-infected individuals were consistently enriched for Butyrivibrio species, and an increase in this species further correlated with a decrease in T-cell activation. These findings argue that some adaptive changes of the gut microbial ecosystem might help alleviate the consequences of the original perturbation (i.e., acute HIV infection) in the intestinal habitat, but others might perpetuate a vicious circle of dysbiosis and immune dysfunction. 

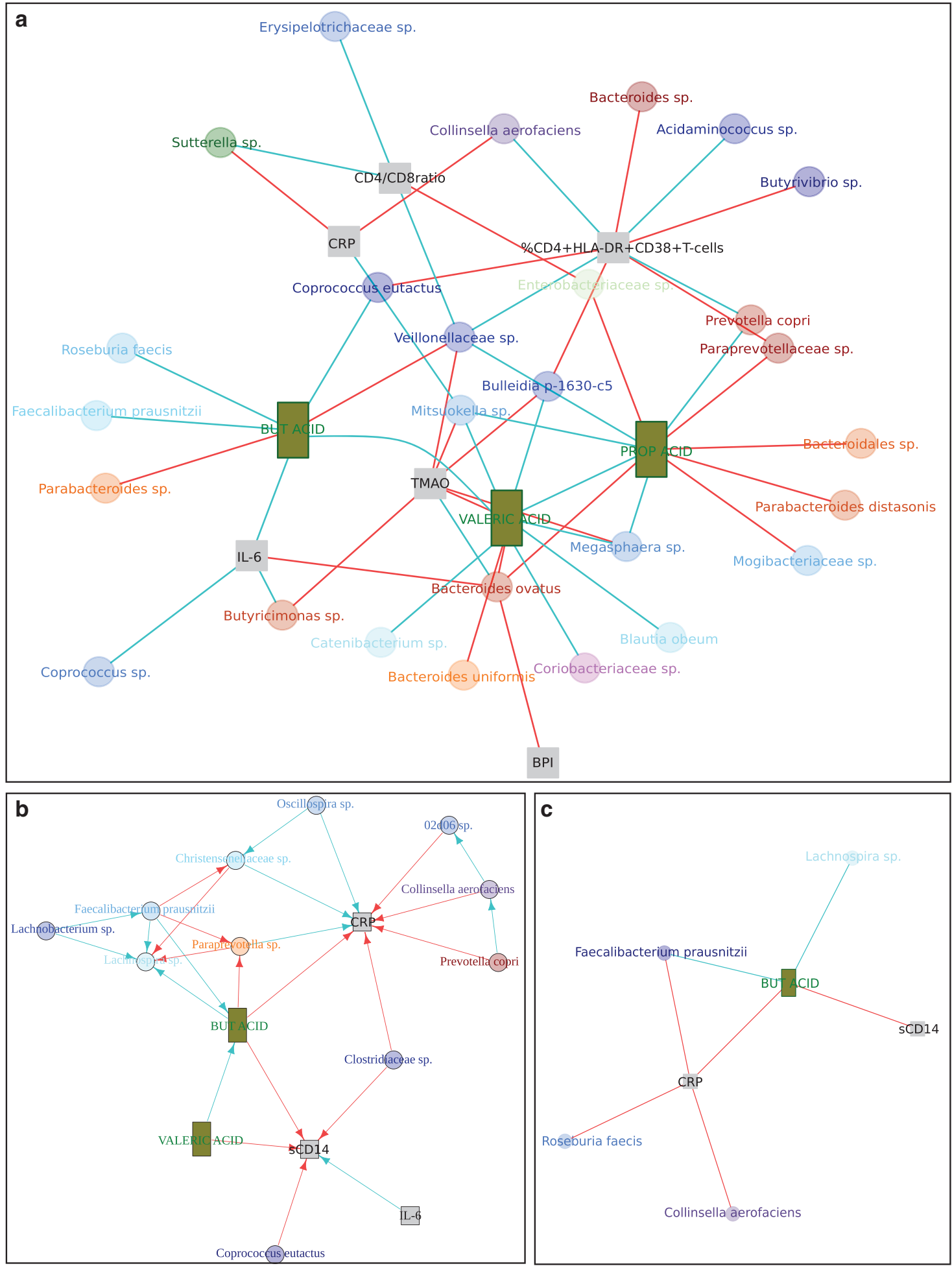

Figure 9 Network integrating genera abundance, SCFA abundance, and markers of innate and adaptive immunity. (a) Interactions between changes in genera contributing to HIV-associated dysbiosis, SCFAs, and peripheral markers of disease progression using generalized linear models are represented in (a). Red edges represent negative correlations, and blue edges positive correlations. Gray squares represent the peripheral markers of disease progression and the green rectangles represent the SCFAs. Blue gradient circles correspond to species to the Firmicutes phylum, red gradient circles correspond to species to the Bacteroidetes, purple gradient correspond to the Actinobacteria phylum and gold gradient correspond to the Fusobacteria phylum. In (a,) the generalized linear model (GLM)-based network of all the $\mathrm{HIV}^{+}$subjects of the cohort and illustrate the interactions between the microbiota, immunological markers and SCFA production are shown. (b, c) Highlight the positive findings in the VU group with the prebiotic intervention by means of a Bayesian Network and a GLM, respectively. Arrows indicate conditional dependencies between variables in the Bayesian Network plotted in (b). The networks ( $\mathbf{b}$ and $\mathbf{c}$ ) highlight the interactions of Faecalibacterium prausnitzii, as well as other known butyrate producers, with butyrate production and the inflammatory markers high-sensitivity C-reactive protein (CRP) and soluble CD14 (SCD14). The increase of the butyrate predicts an increase of species related to SCFA production and a decrease of the inflammatory markers CRP and SCD14. SCFA, short-chain fatty acid. 
As described above, the analysis of Unifrac distances suggested that the microbiota configuration was easier to modulate in VU individuals, who experienced a significant increase in butyrate production. Thus, in this group, we modeled the interactions among the bacterial biomarkers, SCFA production, and the peripheral biomarkers in a Bayesian network, and we estimated the Markov Blanket for the butyrate (Figure 9b). This method is able to infer multiple causal relationships for a given effect. We found that increases in the abundance of Faecalibacterium prausnitzii determined an increase in butyrate, which in turn correlated with a decrease in the levels of two inflammatory predictors of mortality, highsensitivity CRP, and soluble CD14. ${ }^{2}$ Finally, because the analyses repeatedly implicated butyrate as a key SCFA, we aimed to identify the most likely bacterial determinants of butyrate abundance in VU individuals, and we modeled the interaction using generalized linear models. As shown in Figure 9c, generalized linear model analysis identified Faecalibacterium prausnitzii and Lachnospira as major drivers of butyrate abundance.

\section{DISCUSSION}

We herein report a pilot study on HIV-infected individuals analyzing the effects of a nutritional intervention with prebiotics on both gut bacterial composition using nextgeneration sequencing and the impact on the intestinal SCFA abundance in feces. We found evidence of interplay between intestinal microbial ecology and the levels of inflammatory predictors of disease progression and characterized key components of the microbiota. This interaction occurred at least partially via the production of butyrate by fecal bacteria. We showed that the response to prebiotics depends on the stability and resilience of the whole bacterial community, which appears to depend on the control of HIV replication and the extent of ART-mediated immunological restoration. Furthermore, our findings argue that some adaptive changes in the gut microbial ecosystem might serve to alleviate the consequences of the perturbation in the intestinal habitat secondary to acute HIV infection, whereas others might perpetuate a vicious circle of dysbiosis and immune dysfunction.

The human gut microbiota comprises an extremely complex bacterial ecosystem that has co-evolved with its host, establishing entangled symbiotic relationships. Carbohydrates, which are a major component of the human diet, include plantderived polysaccharides (cellulose, hemicellulose, and pectin), starches, and sugars. ${ }^{44}$ Human cells are able to hydrolyze some disaccharides and absorb monosaccharides as glucose or galactose, but they cannot break down most of the polysaccharides. Thus, a considerable number of dietary carbohydrates and proteins pass to the colon after having escaped digestion in the small bowel, and they are then metabolized by the gut microbiota, which contains a large repertoire of hydrolytic enzymes, producing mainly SCFAs. ${ }^{44-47}$ It is now widely accepted that SCFAs have an important role in maintaining health and that they are associated with a wide range of diseases, including inflammatory bowel disease, type 2 diabetes, obesity, celiac disease, allergies, colon cancer, and autoimmune disease. ${ }^{4-50}$ As observed under other proinflammatory conditions, ${ }^{51}$ dietary modulation of gut microbiota with a diet rich in non-digestible but fermentable carbohydrates significantly promoted beneficial groups of bacteria and increased butyrate-producers, specifically the Faecalibacterium prausnitzii and Lachnospira species, which contributed to the alleviation of inflammation in HIV-infected patients,. Hence, the dietary modulation of gut microbiota holds promise as a viable intervention strategy for HIV infection to attenuate persistent immune defects. Our data also suggest, however, that the modulatory capacity of these interventions might vary depending on the fermentative capability of the baseline altered bacterial community and on the type and chemical structure of prebiotics. ${ }^{51}$ For example, whereas GOS are mainly degraded by Actinobacteria (Bifidobacterium, Collinsella), FOS can be metabolized by many microorganisms of different phyla (Firmicutes, Bacteroidetes, and Actinobacteria), and the observed effects would depend on the microbial digestion of each oligosaccharide. ${ }^{44}$

We observed a spectrum of HIV-associated dysbiosis, reflecting the disease status of the study participants and determined by the extent of immunovirological control. Consistent with previous studies in HIV-infected subjects, ${ }^{4,8,33}$ we observed enrichment for Prevotella, a member of the Bacteroidetes phylum that has been linked with heightened T-cell activation in $\mathrm{HIV}^{+}$individuals, ${ }^{8}$ and depletion of Bacteroides. ${ }^{6,10,33}$ In addition, we found decreased levels of butyrate-producers, such as Faecalibacterium, Lachospira and Roseburia, and enrichment of pro-inflammatory genera, such as Succinivibrio, Desulfovibrio and Fusobacterium. We have previously shown in treated HIV-infected individuals that this pattern of dysbiosis is characterized by an enrichment of genes involved in lipopolysaccharide biosynthesis and a depletion of genes involved in amino acid metabolism and energy processes, suggesting that an altered functional profile could contribute to chronic bacterial translocation and immune dysfunction. ${ }^{9}$ In contrast to previous studies in HIV-infected subjects, ${ }^{6,10,52}$ we found decreased levels of Erysipelotrichaceae. Given the strong impact of diet on this family $y^{53}$ and its correlation with obesity, this could have been affected by the Mediterranean diet and normal body mass index that characterized our study population. Still, whether HIV-associated dysbiosis is a result of HIV infection itself or an effect of a combination of several putative drivers, including ART and lifestyle factors, remains debated. ${ }^{32,54,55}$ Indeed, at least two different studies in SIVinfected macaques have failed to detect differences in the intestinal microbiota composition, ${ }^{56-58}$ in striking contrast with human studies, although a recent study of untreated acute SIV infection of non-human primates demonstrated depletion of Lactobacilli, supporting previous findings in HIV-infected individuals. $^{59}$

Because FOS stimulate the growth of bacteria such as the F. prausnitzii, Eubacterium rectale, Roseburia inulinivorans, and Lachnospira species, all of them butyrate-producer species, ${ }^{44,60}$ we expected an increase in these taxa after 
administration of prebiotics. In VU individuals, the nutritional supplement increased the abundance of the depleted Faecalibacterium, a bacteria that is also depleted in Crohn's disease and ulcerative colitis, another inflammatory condition, ${ }^{49,61}$ as well as other genera belonging to the Firmicutes (Catenibacterium, Blautia, Eubacterium) and Actinobacteria (Collinsella and Corinebacterium) phyla. These changes indicate a beneficial impact of the intervention on HIVassociated dysbiosis. In addition to the important role of Faecalibacterium in inducing regulatory $\mathrm{T}$ cells ${ }^{34}$ and decreasing intestinal permeability, ${ }^{62}$ it has been shown that Blautia increases following fecal microbiota transplantation from healthy donors to individuals with recurrent Clostridium difficile infections, who represent a subgroup of individuals with extremely impaired gut bacterial composition, ${ }^{61}$ and Catenibacterium, Eubacterium, and Blautia have been associated with SCFA production. ${ }^{63-67} \mathrm{We}$ also modeled the relationships among the bacteria that most likely influence the maintenance of chronic immunological abnormalities and found evidence that butyrate-producer species, particularly the fiber-fermenter and SCFA producers Faecalibacterium prausnitzii and Lachnospira species, might be viable targets for interventions. Although we did detect a significant increase in the butyrate levels after the prebiotic intervention in the VU group, it should be noted that no significant differences were noted across groups at baseline. The fact that the butyrate concentrations were one-third lower in VU than in the other groups suggests that this could be explained by insufficient statistical power to detect changes at this level.

The effects on the microbiota composition were less apparent among treated $\mathrm{HIV}^{+}$individuals, yet we detected some significant effects on markers of innate and adaptive immunity in peripheral blood (i.e., a decrease in BPI, decreased $\mathrm{T}$-cell activation, improved thymic output, and changes in host gene expression). The younger median age of the VU group might have influenced in the higher impact of the nutritional intervention in the bacterial community in this group; we think, however, that a more compelling explanation is related to the longer median exposure to HIV disease of ART-treated participants (IR, 8.9 years; INR, 6.1 years) compared with that of VU individuals (5.4 years). We suggest a model, based on ecological principles, in which shorter exposure to the HIV disease might have elicited dysbiosis with a degraded transient state of the bacterial community. The combined and perhaps synergistic effects of a prolonged situation of chronic immunologic dysfunction, and ART itself, could result in a secondary transition of microbiota structure to a degraded stable state capable of resisting colonization by the presumably beneficial species selected by the prebiotics. ${ }^{68,69}$ Based on this model, only the VU-associated microbiota, in a transient state and with decreased resistance to an external nutritional intervention, was able to clearly respond to the prebiotics. Although the landscape of stable states for the human gut is far from being determined, these findings suggest that HIVassociated dysbiosis might achieve a stable configuration over time and that the dysbiosis of patients who have lived with HIV for many years might prove more difficult to reverse than that of their counterparts.

Some limitations must be considered when interpreting our results. First, this is a pilot study, and the limited sample size warrants caution regarding the conclusions. We measured many parameters in small groups, so we cannot rule out that some of the statistically significant changes are due to randomness rather than the intervention. In addition, the small number of patients receiving the placebo was useful in assessing the tolerability of the intervention but insufficient for comparisons of the assessed markers among arms. Because this study concluded before the effect of sexual orientation on the microbiota was appreciated, ${ }^{32}$ we had to conduct post hoc analyses to control for this potential confounder. Second, a limitation of most studies trying to understand the effects of shaping the microbiota, including ours, is that nutritional interventions could alter bacterial metabolism, which was not measured in our study, without altering bacterial composition. Third, SCFA abundance was measured in feces, so the measurements represent the difference between production of SCFAs by the bacterial community and their uptake by the gut mucosa. Fourth, we measured bacterial translocation using only indirect markers (i.e., BPI and soluble CD14), and we did not sample the intestinal mucosa, which would have provided further insight into the bacterial communities driving mucosal disruption, immune defects, and increased gut permeability, so we cannot provide experimental evidence that the intervention affected gut immunity. Finally, the study was not designed to specifically assess the effect of dietary changes on gut microbiota beyond the prebiotic intervention, and limited information on diet was collected. Therefore, given the strong dependence of TMAO on diet, it is difficult to interpret the significant increase of this metabolite that was observed in the IR group. ${ }^{38}$

The field has only begun to scratch the surface of the potential implications of HIV-associated dysbiosis in the maintenance of immune dysfunction, and the study of gut microbiota modulation in this setting is in its early stages. So far, it is unclear how to best shape the gut microbiome to modulate immunity. Although influencing the microbiota with a probiotic might prove harder than doing so with a prebiotic, studies comparing the effects of different probiotic strains and dietary interventions are lacking. There is, however, emerging consensus that a number of bacterial-derived metabolites, particularly SCFA, can impact systemic metabolism and immunity. Our study expands previous knowledge on the effects of microbiome-targeted interventions in $\mathrm{HIV}^{+}$individuals by a comprehensive analysis of the influence of changes in gut bacterial composition and in SCFA production on innate and adaptive immunity. We show that a short and well-tolerated nutritional intervention influenced gut microbiota structure and elicited downstream effects on a number of markers of clinical progression, although its impact would depend on the stability of the gut microbial community. Our data show a distinct intestinal microbial makeup related to the extent of immunovirological control and suggest that the gut microbiota is a viable target for interventions. Along with recent studies using other nutritional interventions aiming to affect gut microbiota, ${ }^{22,23}$ our data encourage testing 
new strategies aiming to affect HIV-associated dysbiosis. Future trials should focus on patients undergoing stable ART and analyze the long-term effects of therapies aiming to ameliorate HIV-associated dysbiosis. Metagenomic and metabolomic approaches and personalized therapies based on microbiota structure would help to understand how we might exploit microbial-host interactions to restore normal health to individuals living with HIV.

\section{METHODS}

Study design, participants, setting, and eligibility. To capture a wide spectrum of HIV immunopathogenesis, we recruited VU HIV-infected subjects, immunological ART responders and non-responders (IR and INR, $\geqslant 350$ and $<350$ CD4 + T-cell counts after $>2$ years of viral suppression, respectively), and unmatched HIV-uninfected $\left(\mathrm{HIV}^{-}\right)$individuals. From the 95 individuals screened in the study, 35 were not eligible, and 60 were randomly assigned to the prebiotic intervention and placebo groups (2:1). A total of 44 individuals completed the 6-week study on treatment, 34 in the active arm $(20 \mathrm{~g}$ mixture of prebiotics, including $5 \mathrm{~g}$ of short-chain galacto-oligosaccharides (Purimune), $10 \mathrm{~g}$ of longchain fructo-oligosaccharides (Orafti-HP and Actilight), and $5 \mathrm{~g}$ of glutamine (Nutrición Médica)) and 10 in the placebo arm (20 g of maltodextrin). The groups were composed of $12 \mathrm{HIV}^{+}$ART subjects ( 9 and 3 in the active and placebo arms, respectively), eight INR subjects (eight and zero), 15 IR subjects (10 and 5), and nine control subjects (seven and two).

Nine patients dropped out of the study (three in the active arm and six in the placebo arm; Figure 1). This study was approved by the Ethics Committees of both recruiting institutions (University Hospital Clínico San Carlos [approval number 11/284], ceic.hcsc@salud.madrid.org, and University Hospital Ramón y Cajal, ceic.hrc@salud.madrid.org). All the participants gave informed consent before initiation of the study procedures. The Clinical Trials Registry Identification Number Identifier (clinicaltrials.gov) for this study is NCT01838915.

Nucleic acid purification, amplification of the 16S rRNA gene, and pyrosequencing. Fecal samples were stored in RNAlater (Life Technologies, Carlsbad, CA) at $-80^{\circ} \mathrm{C}$ until use. Total DNA was extracted as described. ${ }^{70}$ Briefly, the samples were diluted (half dilution) in phosphate-buffered saline (PBS). Then, they were centrifuged at 2,000 r.p.m. at $4{ }^{\circ} \mathrm{C}$ for $2 \mathrm{~min}$ to remove fecal debris. Total DNA was extracted from the pelleted cells with QIAamp DNA Stool Kit (Qiagen, Hilden, Germany) according to the manufacturer's instructions. For each sample, a region of the $16 \mathrm{~S}$ rRNA gene was amplified by polymerase chain reaction (PCR) with the universal primers E8F (5'-AGAGTTTGATCMTGGCTCAG-3') and 530R $\left(5^{\prime}\right.$-CCGCGGCKGCTGGCAC-3'). The E8F primer included a sample-specific Multiplex Identifier (MID) to be multiplexed and sequenced simultaneously. The pooled PCR products were directly pyrosequenced. The $16 \mathrm{~S}$ rRNA gene amplicons were pyrosequenced using a Roche GS FLX sequencer and Titanium chemistry in the Centre for Public Health Research (FISABIO-Salud Pública, Valencia, Spain). All the sequences were deposited in the public European Nucleotide Archive server under accession number PRJEB5185. 16S RNA phylogenetic analysis, biodiversity, clustering, and biomarker discovery analysis are described in the SM.

\section{Systemic biomarkers of disease progression}

Markers of innate immune activation and bacterial translocation. A sample of fasting venous blood was obtained to determine the concentrations of glucose, interleukin-6, plasma HIV RNA, highsensitivity CRP, D-dimers, BPI, and soluble CD14 as described in the SM.
Markers of adaptive immune activation. T-cell immunophenotyping: Fresh EDTA-anticoagulated whole blood was used to quantify the percentage of $\mathrm{CD}^{+}$and $\mathrm{CD}^{+}{ }^{+} \mathrm{T}$ cells expressing markers of activation $\left(\mathrm{CD}_{2} 5^{+}, \mathrm{CD}_{3} 8^{+}, \mathrm{HLADR}^{+}\right.$or $\left.\mathrm{CD} 38^{+} / \mathrm{HLA}^{-\mathrm{DR}^{+}}{ }^{+}\right)$and senescence $\left(\mathrm{CD} 7^{+}\right)$by flow cytometry as previously described ${ }^{9}$ and detailed in the SM.

Quantification of sj/ $\beta$-TREC ratio: Thymic function was indirectly calculated from the DNA of peripheral blood mononuclear cells (PBMCs) using the sj/ $\beta$-TREC ratio previously described ${ }^{71}$ with minor modifications, as detailed in the SM.

Short-chain fatty acids. We used the bacterial suspension obtained as described above. The bacterial fraction $(455 \mu \mathrm{l})$ containing 4-methyl valeric acid as an internal standard ( $500 \mu \mathrm{m}$ final concentration) was acidified with $45 \mu \mathrm{l}$ of orthophosphoric acid ( $9 \%$ final concentration) and maintained for $10 \mathrm{~min}$ with continuous shaking to facilitate the liberation of SCFAs from the fecal matrix. A detailed description of the method is provided in the SM.

Statistical methods and bioinformatics analysis. Although the study is a pilot trial, the sample size of the intervention chosen to ensure adequate power to estimate the effect size was based on that of a previous controlled study. ${ }^{18}$ Previous studies in model animals and humans have shown detectable effects with prebiotics after 6 weeks of treatment. $^{18,72-75}$

To evaluate differences in the continuous variables across groups, we used the Kruskal-Wallis test. Between-group comparisons of continuous variables were analyzed using Wilcoxon rank-sum test. For the microbiota analysis, differences in Shannon diversity index and richness estimators (Chaol and ACE) were analyzed using the same test.

We used linear mixed models with a random effect for each patient to allow for correlations caused by repeated observations to assess whether longitudinal changes in numerical outcome measures were overall significantly different from baseline. A robust variance estimator was used, given the limited sample size and the deviations from normality. Continuous variables in mixed models were logtransformed when necessary to satisfy model assumptions. Given the low number of individuals receiving placebo, to assess the efficacy of the intervention on peripheral markers, we calculated the statistical significance for the change of each parameter after the intervention in the active group.

The methods that were used to model the interactions between changes in genera contributing to HIV-associated dysbiosis changes in peripheral markers of disease progression and changes in SCFA abundance using generalized linear models and Bayesian networks are provided in the Supplementary Material online, as are the methods used to perform permutation tests and construct rarefaction curves.

Given the high-dimensional data and to control the false discovery rate, we validated the statistical tests using the following strategies. First, all $P$ values were adjusted using the Benjamini-Hochberg correction (library 'stats', function 'p.adjust'). Second, we estimated the power (R library 'pwr' functions: 'power.anova.test' and 'power.t.test') of each of the statistical test to verify the robustness of the results (Supplementary Table S9). Third, we performed permutation tests (Supplementary Table S9), which are increasingly used when the sample size is low or when the distribution of data is unknown. Permutation tests are able to construct sampling distributions by resampling the observed data. These analyses were performed using the $\mathrm{R}$ package, coin library, and pwr.anova.test and pwr.t.test functions.

Statistical and bioinformatic analyses were conducted with Stata v. 12.0 (StataCorp LP, College Station, TX, USA) and R statistical software version 3.2.0.

SUPPLEMENTARY MATERIAL is linked to the online version of the paper at http://www.nature.com/mi 


\section{ACKNOWLEDGMENTS}

We acknowledge the participation of all the study participants who contributed to this work as well as the clinical research staff of the participating institutions who made this research possible. We thank Francisco Martín-Azcárate and Pablo Manzano from the Ecology Department, of Autómoma University of Madrid, for helpful discussions on ecosystem dynamics, Ester Domínguez for her technical support at University Hospital Ramón y Cajal and Nuria Jiménez for her laboratory and pyrosequencing work in the Genomics and Health Area of FISABIO-Public Health Institute of Valencia. This work was supported by the Instituto de Salud Carlos III (Plan Estatal de I+D + i 2013-2016, projects PI13/00438, PIE14/00045, PI15/00345 and EE) and cofinanced by the European Development Regional Fund "A way to achieve Europe" (ERDF), Health Research Institute of University Hospital Clínico San Carlos, "Generalitat Valenciana" (Prometeo/2009/092) and by the Spanish Ministry of Economy and Competitiveness (SAF2012-31187 and CTQ2014-55279-R), Redes Temáticas de Investigación en SIDA (ISCIII RETIC RD12/0017/0029 and RD12/0017/0037, RIS-EST37), and Junta de Andalucia (Proyecto de Excelencia CTS-6313). S.S.-V. is supported by a grant from the Spanish Ministry of Science and Innovation (Contratos Juan Rodés, ECC/1051/ 2013). J.F.V.-C. was supported by a fellowship "Ayudas Predoctorales de Formación en Investigación en Salud" from the Instituto de Salud Carlos III, Spain and by a fellowship "Ayudas Predoctorales de Formación en Investigación en Salud" from the Instituto de Salud Carlos III, Spain and the CONACYT-SECITI fellowship, México; and T.S. by a grant from the European Society of Pediatric Infectious Diseases (ESPID).

\section{AUTHOR CONTRIBUTIONS}

S.S.-V., M.J.G., and V.E. designed the study and experiments; J.F.V.-C. and M.J.G. performed the metagenomic experiments and bioinformatic analyses; A.L. and A.M. supervised the metagenomic analyses; A.V. performed the flow-cytometry experiments; N.M. measured plasma biomarkers; J.M.-B. performed the gene expression experiments; S.F.$M$ and M.L. performed the thymic function experiments; D.R. and C.B. performed the mass spectrometry experiments. S.S.-V. obtained institutional review board approval; S.S.-V, T.S., J.d.R., S.M., and V.E. managed study participant recruitment; S.S.-V. and J.F.V.-C. analyzed the data on systemic markers. S.S.-V., J.F.V.-C., and M.J.G wrote the first version of the manuscript. M.F. provided a critical revision of the manuscript. All the authors reviewed and approved the manuscript.

\section{DISCLOSURE}

The authors declared no conflict of interest.

c) 2017 Society for Mucosal Immunology

\section{REFERENCES}

1. Klatt, N.R., Chomont, N., Douek, D.C. \& Deeks, S.G. Immune activation and HIV persistence: implications for curative approaches to HIV infection. Immunol. Rev. 254, 326-342 (2013).

2. Hunt, P.W. et al. Gut epithelial barrier dysfunction and innate immune activation predict mortality in treated HIV infection. J. Infect. Dis. 210, 1228-1238 (2014).

3. Sandler, N.G. \& Douek, D.C. Microbial translocation in HIV infection: causes, consequences and treatment opportunities. Nat. Rev. Microbiol. 10, 655-666 (2012).

4. Ling, Z. et al. Alterations in the fecal microbiota of patients with HIV-1 infection: an observational study in a Chinese population. Sci. Rep 6 , 30673 (2016).

5. Pérez-Santiago, J., Gianella, S. \& Massanella, M. Gut lactobacillales are associated with higher CD4 and less microbial translocation during HIV infection. AIDS 27, 1921-1931 (2013).

6. Lozupone, C. et al. Alterations in the gut microbiota associated with HIV-1 infection. Cell Host Microbe 14, 329-339 (2013).

7. McHardy, I.H. et al. HIV infection is associated with compositional and functional shifts in the rectal mucosal microbiota. Microbiome 1, 26 (2013).
8. Dillon, S.M. et al. An altered intestinal mucosal microbiome in HIV-1 infection is associated with mucosal and systemic immune activation and endotoxemia. Mucosal Immunol 7, 983-994 (2014).

9. Vázquez-Castellanos, J.F. et al. Altered metabolism of gut microbiota contributes to chronic immune activation in HIV-infected individuals. Mucosal Immunol 8, 760-772 (2015).

10. Vujkovic-Cvijin, I. et al. Dysbiosis of the gut microbiota is associated with HIV disease progression and tryptophan catabolism. Sci. Transl. Med. 5, $193 r a 91$ (2013).

11. Nowak, P. et al. Gut microbiota diversity predicts immune status in HIV-1 infection. AIDS 29, 2409-2418 (2015).

12. Favre, D. et al. Tryptophan catabolism by indoleamine 2,3-dioxygenase 1 alters the balance of TH17 to regulatory T cells in HIV disease. Sci. Transl. Med. 2, 32 ra36 (2010).

13. Chang, P.V, Hao, L., Offermanns, S. \& Medzhitov, R. The microbial metabolite butyrate regulates intestinal macrophage function via histone deacetylase inhibition. Proc. Natl. Acad. Sci. USA 111, 2247-2252 (2014).

14. Louis, P. \& Flint, H.J. Diversity, metabolism and microbial ecology of butyrate-producing bacteria from the human large intestine. FEMS Microbiol. Lett. 294, 1-8 (2009).

15. Serrano-Villar, S. et al. Gut bacteria metabolism impacts immune recovery in HIV-infected individuals. EBioMedicine 8, 203-216 (2016).

16. Bik, E.M. Composition and function of the human-associated microbiota. Nutr. Rev. 67 (Suppl 2), S164-S171 (2009).

17. Burger-van Paassen, N. et al. The regulation of intestinal mucin MUC2 expression by short-chain fatty acids: implications for epithelial protection. Biochem. J. 420, 211-219 (2009).

18. Gori, A. et al. Specific prebiotics modulate gut microbiota and immune activation in HAART-naive HIV-infected adults: results of the 'COPA' pilot randomized trial. Mucosal Immunol 4, 1-10 (2011).

19. Hummelen, R., Vos, A.P., van't Land, B., Norren, K. \& van Reid, G. Altered host-microbe interaction in HIV: a target for intervention with pro- and prebiotics. Int. Rev. Immunol. 29, 485-513 (2010).

20. Schunter, M. et al. Randomized pilot trial of a synbiotic dietary supplement in chronic HIV-1 infection. BMC Complement Altern. Med. 12, 84 (2012).

21. Cahn, P. et al. The immunomodulatory nutritional intervention NR100157 reduced CD4 + T-cell decline and immune activation: a 1-year multicenter randomized controlled double-blind trial in HIV-infected persons not receiving antiretroviral therapy (The BITE Study). Clin. Infect. Dis. 57, 139-146 (2013).

22. Villar-García, J. et al. Effect of probiotics (Saccharomyces boulardii) on microbial translocation and inflammation in HIV-treated patients: a doubleblind, randomized, placebo-controlled trial. J. Acquir. Immune Defic. Syndr. 68, 256-263 (2015).

23. Stiksrud, B. et al. Reduced levels of D-dimer and changes in gut microbiota composition after probiotic intervention in HIV-infected individuals on stable ART. J. Acquir. Immune Defic. Syndr. 70, 329-337 (2015).

24. González-Hernández, L.A. et al. Synbiotic therapy decreases microbial translocation and inflammation and improves immunological status in HIV-infected patients: a double-blind randomized controlled pilot trial. Nutr. J. 11, 90 (2012).

25. Hummelen, R. et al. Effect of 25 weeks probiotic supplementation on immune function of HIV patients. Gut Microbes 2, 80-85 (2011).

26. Klatt, N.R. et al. Probiotic/prebiotic supplementation of antiretrovirals improves gastrointestinal immunity in SIV-infected macaques. J. Clin. Invest. 123, 903-907 (2013).

27. Wang, L. et al. Dietary supplementation with glutamate precursor $\alpha$ ketoglutarate attenuates lipopolysaccharide-induced liver injury in young pigs. Amino Acids 47, 1309-1318 (2015).

28. Pai, M.-H., Shih, Y.-M., Shih, J.-M. \& Yeh, C.-L. Glutamine modulates changes in intestinal intraepithelial $\gamma \delta T$ lymphocyte expressions in mice with ischemia/reperfusion injury. Shock 44, 77-82 (2015).

29. Moore, S.R. et al. Glutamine and alanyl-glutamine promote crypt expansion and mTOR signaling in murine enteroids. Am. J. Physiol. Gastrointest. Liver Physiol. 308, G831-G839 (2015).

30. O'Flaherty, S., Saulnier, D. M., Pot, B. \& Versalovic, J. How can probiotics and prebiotics impact mucosal immunity?. Gut Microbes 1, 293-300 (2010). 
31. De-Souza, D. A. \& Greene, L. J. Intestinal permeability and systemic infections in critically ill patients: effect of glutamine. Crit. Care Med. 33, 1125-1135 (2005).

32. Noguera-Julian, M. et al. Gut microbiota linked to sexual preference and HIV infection. EBioMedicine 5, 135-146 (2016).

33. Mutlu, E.A et al. A compositional look at the human gastrointestinal microbiome and immune activation parameters in HIV infected subjects. PLoS Pathog. 10, e1003829 (2014).

34. Sarrabayrouse, G. et al. CD4CD8 $\alpha \alpha$ lymphocytes, a novel human regulatory $\mathrm{T}$ cell subset induced by colonic bacteria and deficient in patients with inflammatory bowel disease. PLoS Biol. 12, e1001833 (2014).

35. Tremaroli, V. \& Bäckhed, F. Functional interactions between the gut microbiota and host metabolism. Nature 489, 242-249 (2012).

36. Somsouk, M. et al. Gut epithelial barrier and systemic inflammation during chronic HIV infection. AIDS 2, 43-51 (2014).

37. Bleijerveld, O.B. et al. Deep proteome profiling of circulating granulocytes reveals bactericidal/permeability-increasing protein as a biomarker for severe atherosclerotic coronary stenosis. J. Proteome Res. 11, 5235-5244 (2012).

38. Tang, W.H.W. et al. Intestinal microbial metabolism of phosphatidylcholine and cardiovascular risk. N. Engl. J. Med. 368, 1575-1584 (2013).

39. Hunt, P.W. et al. Impact of CD8 + T-cell activation on CD4 + T-cell recovery and mortality in HIV-infected Ugandans initiating antiretroviral therapy. AIDS 25, 2123-2131 (2011).

40. Li, T. et al. Reduced thymic output is a major mechanism of immune reconstitution failure in HIV-infected patients after long-term antiretroviral therapy. Clin. Infect. Dis. 53, 944-951 (2011).

41. Ridaura, V.K. et al. Gut microbiota from twins discordant for obesity modulate metabolism in mice. Science 341, 1241214 (2013).

42. Allers, K. et al. Macrophages accumulate in the gut mucosa of untreated HIV-infected patients. J. Infect. Dis. 209, 739-748 (2013).

43. Pak-Wittel, M.A., Yang, L., Sojka, D.K., Rivenbark, J.G. \& Yokoyama, W.M. Interferon- $\gamma$ mediates chemokine-dependent recruitment of natural killer cells during viral infection. Proc. Natl. Acad. Sci. USA 110, E50-E59 (2013).

44. Flint, H.J., Scott, K.P., Louis, P. \& Duncan, S.H. The role of the gut microbiota in nutrition and health. Nat. Rev. Gastroenterol. Hepato/ 9, 577589 (2012).

45. Hooper, L.V, Midtvedt, T. \& Gordon, J.I. How host-microbial interactions shape the nutrient environment of the mammalian intestine. Annu. Rev. Nutr. 22, 283-307 (2002).

46. Gill, S.R. et al. Metagenomic analysis of the human distal gut microbiome. Science 312, 1355-1359 (2006).

47. Qin, J. et al. A human gut microbial gene catalogue established by metagenomic sequencing. Nature 464, 59-65 (2010).

48. Sokol, $\mathrm{H}$. et al. Faecalibacterium prausnitzii is an anti-inflammatory commensal bacterium identified by gut microbiota analysis of Crohn disease patients. Proc. Natl. Acad. Sci. USA 105, 16731-16736 (2008).

49. Machiels, K. etal. A decrease of the butyrate-producing species Roseburia hominis and Faecalibacterium prausnitzii defines dysbiosis in patients with ulcerative colitis. Gut 63, 1275-1283 (2014).

50. Carding, S., Verbeke, K., Vipond, D.T., Corfe, B.M. \& Owen, L.J. Dysbiosis of the gut microbiota in disease. Microb. Ecol. Health Dis 26, 26191 (2015).

51. Zhang, C. et al. Dietary modulation of gut microbiota contributes to alleviation of both genetic and simple obesity in children. EBioMedicine 2 , 968-984 (2015).

52. Sun, Y. et al. Fecal bacterial microbiome diversity in chronic HIV-infected patients in China. Emerg. Microbes Infect. 5, e31 (2016).

53. Kim, M.S., Hwang, S.S., Park, E.J. \& Bae, J.W. Strict vegetarian diet improves the risk factors associated with metabolic diseases by modulating gut microbiota and reducing intestinal inflammation. Environ. Microbiol. Rep 5, 765-775 (2013).
54. Lozupone, C.A. et al. HIV-induced alteration in gut microbiota: driving factors, consequences, and effects of antiretroviral therapy. Gut Microbes 5, 562-570 (2014).

55. Williams, B., Landay, A. \& Presti, R.M. Microbiome alterations in HIV infection a review. Cell. Microbiol. 18, 645-651 (2016).

56. Klatt, N.R., Funderburg, N.T. \& Brenchley, J.M. Microbial translocation, immune activation, and HIV disease. Trends Microbiol. 21, 6-13 (2013).

57. Handley, S.A. et al. Pathogenic simian immunodeficiency virus infection is associated with expansion of the enteric virome. Cell 151, 253-266 (2012).

58. Barbian, H.J. et al. Destabilization of the gut microbiome marks the endstage of simian immunodeficiency virus infection in wild chimpanzees. Am. J. Primatol. (2015); http://onlinelibrarywiley.com/wol1/doi/10.1002/ ajp.22515/full.

59. Vujkovic-Cvijin, I. et al. Gut-resident Lactobacillus abundance associates with IDO1 inhibition and Th17 dynamics in SIV-infected macaques. Cell Rep 13, 1589-1597 (2015).

60. Louis, P., Scott, K.P., Duncan, S.H. \& Flint, H.J. Understanding the effects of diet on bacterial metabolism in the large intestine. J. Appl. Microbiol. 102, 1197-1208 (2007).

61. Seekatz, A.M. et al. Recovery of the gut microbiome following fecal microbiota transplantation. MBio 5, e00893-14 (2014).

62. Laval, L. et al. Lactobacillus rhamnosus CNCM I-3690 and the commensal bacterium Faecalibacterium prausnitzii A2-165 exhibit similar protective effects to induced barrier hyper-permeability in mice. Gut Microbes 6, 1-9 (2015).

63. Zhang, C. et al. Interactions between gut microbiota, host genetics and diet relevant to development of metabolic syndromes in mice. ISME J. 4, 232 241 (2010).

64. Moya, A. \& Ferrer, M. Functional redundancy-induced stability of gut microbiota subjected to disturbance. Trends Microbiol. 24, 402-413 (2016).

65. Kageyama, A. \& Benno, Y. Catenibacterium mitsuokai gen. nov., sp. nov., a Gram-positive anaerobic bacterium isolated from human faeces $50 \mathrm{Pt} 4$, 1595-1599 (2016).

66. Schwiertz, A., Lehmann, U., Jacobasch, G. \& Blaut, M. Influence of resistant starch on the SCFA production and cell counts of butyrateproducing Eubacterium spp. in the human intestine. J. Appl. Microbiol. 93, 157-162 (2002).

67. den Besten, G. et al. Gut-derived short-chain fatty acids are vividly assimilated into host carbohydrates and lipids. Am. J. Physiol. Gastrointest. Liver Physiol. 305, G900-G910 (2013).

68. Holling, C.S. Resilience and stability of ecological systems. Annu. Rev. Ecol. Syst. 4, 1-23 (1973).

69. Lozupone, C., Stombaugh, J.I., Gordon, J.I., Jansson, J.K. \& Knight, R. Diversity, stability and resilience of the human gut microbiota. Nature 489 , 220-230 (2012)

70. Pérez-Cobas, A.E. et al. Gut microbiota disturbance during antibiotic therapy: a multi-omic approach. Gut 62, 1591-1601 (2013).

71. Ferrando-Martínez, S. et al. A reliable and simplified sj/beta-TREC ratio quantification method for human thymic output measurement. J. Immunol. Methods 352, 111-117 (2010).

72. Liu, Z. et al. Prebiotic effects of almonds and almond skins on intestinal microbiota in healthy adult humans. Anaerobe 26, 1-6 (2014).

73. Shukla, G. Prebiotic inulin alters the colonic mass, $\mathrm{pH}$, microflora and short chain fatty acids in 1,2-dimethylhydrazine dihydrochloride induced early colon carcinogenesis in male Laca mice. J. Probiotics Health 2, 1-6 (2014).

74. Eberhard, M. et al. Effect of inulin supplementation on selected gastric, duodenal, and caecal microbiota and short chain fatty acid pattern in growing piglets. Arch. Anim. Nutr. 61, 235-246 (2007).

75. Ketabi, A., Dieleman, L.A. \& Gänzle, M.G. Influence of isomaltooligosaccharides on intestinal microbiota in rats. J. Appl. Microbiol. 110, 1297-1306 (2011). 